\title{
Regionalization and Intersegmental Coordination of Rhythm- generating Networks in the Spinal Cord of the Chick Embryo
}

\author{
Stephen Ho and Michael J. O'Donovan \\ Section on Developmental Neurobiology, Laboratory of Neural Control, NINDS, NIH, Bethesda, Maryland 20892
}

We have examined the regionalization and coordination of rhythm-generating networks in the isolated spinal cord of the chick embryo between embryonic days 9 and 13, by recording the pattern of rhythmic activity recorded from muscle nerves and ventral roots following a variety of lesions.

We found that the capacity for rhythmic activity is distributed along the rostrocaudal axis of the cord but can be expressed in a single, isolated segment. Specializations within the lumbosacral cord were investigated by isolating particular regions and recording their motor output. The rostral part of the lumbosacral cord generates more cycles than the caudal part, and this difference becomes more pronounced with development.

In the unlesioned cord, motoneuron activity is synchronized along the rostrocaudal axis. Lesion experiments revealed that the synchronization of motoneuron activity and the synaptic drive to caudal motoneurons is mediated in part by propriospinal pathways traveling in the ventrolateral white matter tracts and by synaptic interactions within the gray matter. The dorsal fiber tracts may also be involved but their effects appear to be weak.

Lesions in dorsal-ventral and mediolateral planes were used to localize regions critical for rhythmogenesis and for the alternation of flexor and extensor motoneurons. Rhythmic activity with alternation persisted in spinal cords in which the dorsal and medial half had been removed. Severe medial or dorsal lesions, resulting in a thin strip of lateral or ventral gray matter, altered the phasing of motoneuron activity from alternating to synchronous without effects on cycle timing. These results suggest that the critical neural components for alternation are located close to and dorsomedial to the lateral motor column, and that the capacity for rhythmogenesis is distributed widely throughout the ventral gray matter and is not localized to specific nuclei.

[Key words: central pattern generator, development, spinal networks, intersegmental coordination, motor activity, spinal regionalization]

Spontaneous neural activity plays an important role in neural development and can influence gene expression, the formation of connections, and neuronal differentiation (Pittman and Oppenheim, 1978; Srihari and Vrbova, 1978; McLennan, 1983;

\footnotetext{
Received June 5, 1992; revised Sept. 18, 1992; accepted Sept. 25, 1992.

We thank Dr. Alan Roberts and Dr. Robert Burke for their comments on the manuscript.

Correspondence should be addressed to Michael O'Donovan, Room 5a29, Building 36, NIH, Bethesda, MD 20892.

Copyright (C 1993 Society for Neuroscience $0270-6474 / 93 / 131354-18 \$ 05.00 / 0$
}

Mattson, 1988; Agoston et al., 1991; Mattson and Hauser, 1991; Meier et al., 1991). In the embryonic chick, rhythmic motor activity emerges early in development and is generated centrally within the spinal cord (Hamburger and Balaban, 1963; Hamburger et al., 1966; Hamburger, 1973; Bekoff et al., 1975; Oppenheim, 1975; Provine and Rogers, 1977; Corner et al., 1979; Landmesser and O'Donovan, 1984; O'Donovan, 1989a). This activity is synchronized along the rostrocaudal axis of the cord both in ovo and in isolated, perfused preparations of the spinal cord (Provine, 1971; Ripley and Provine, 1972; Landmesser and O'Donovan, 1984; O'Donovan and Landmesser, 1987). At present, the neural mechanisms responsible for the genesis of rhythmic activity and its synchronization along the cord are unknown.

Intracellular and whole-cell patch-clamp recordings from motoneurons have suggested that rhythmic motoneuron activity is produced by a network of premotor interncurons (O'Donovan, 1989a; Sernagor and O'Donovan, 1991; O'Donovan et al., 1992), but the precise location and composition of these networks are unknown. A critical first stage in understanding the organization of these networks requires their localization and the identification of their constituent interneurons. Previous studies of motor activity in ovo suggest that some components of these networks may be located in the ventral part of the spinal cord. This conclusion is based on the observations that spontaneous neural activity is most prominent in ventral neurons (Sharma et al., 1970; Provine, 1971) and embryonic movements persist in spinal cords that have had the dorsal part removed early in development (Hamburger et al., 1966). It is not known, however, if such lesions disrupt the normal timing or phasing of motoneuron activity, because electromyographic recordings were not performed on the dorsally lesioned animals.

In this article we have investigated the location of rhythmgenerating networks and their synchronization along the spinal cord by documenting the effect of selective lesions on the pattern of motor output recorded from muscle nerves and ventral roots. We were particularly interested in establishing if rhythm generation in the chick cord is localized to a particular region or nuclear group within the lumbosacral cord. In some vertebrates there is evidence for specialization of the rhythm-generating circuitry within the hindlimb enlargement of the spinal cord. In both the cat (Deliagina et al., 1983; Arshavsky et al., 1984; Gelfand et al., 1988) and the turtle (Mortin and Stein, 1989), the anterior segments of the enlargement have a greater capacity for generating rhythmic scratching than the posterior segments. Our results show that the anterior segments of the lumbosacral cord of the embryonic chick are more capable than caudal segments of generating the rhythmic motor activity and that rhyth- 

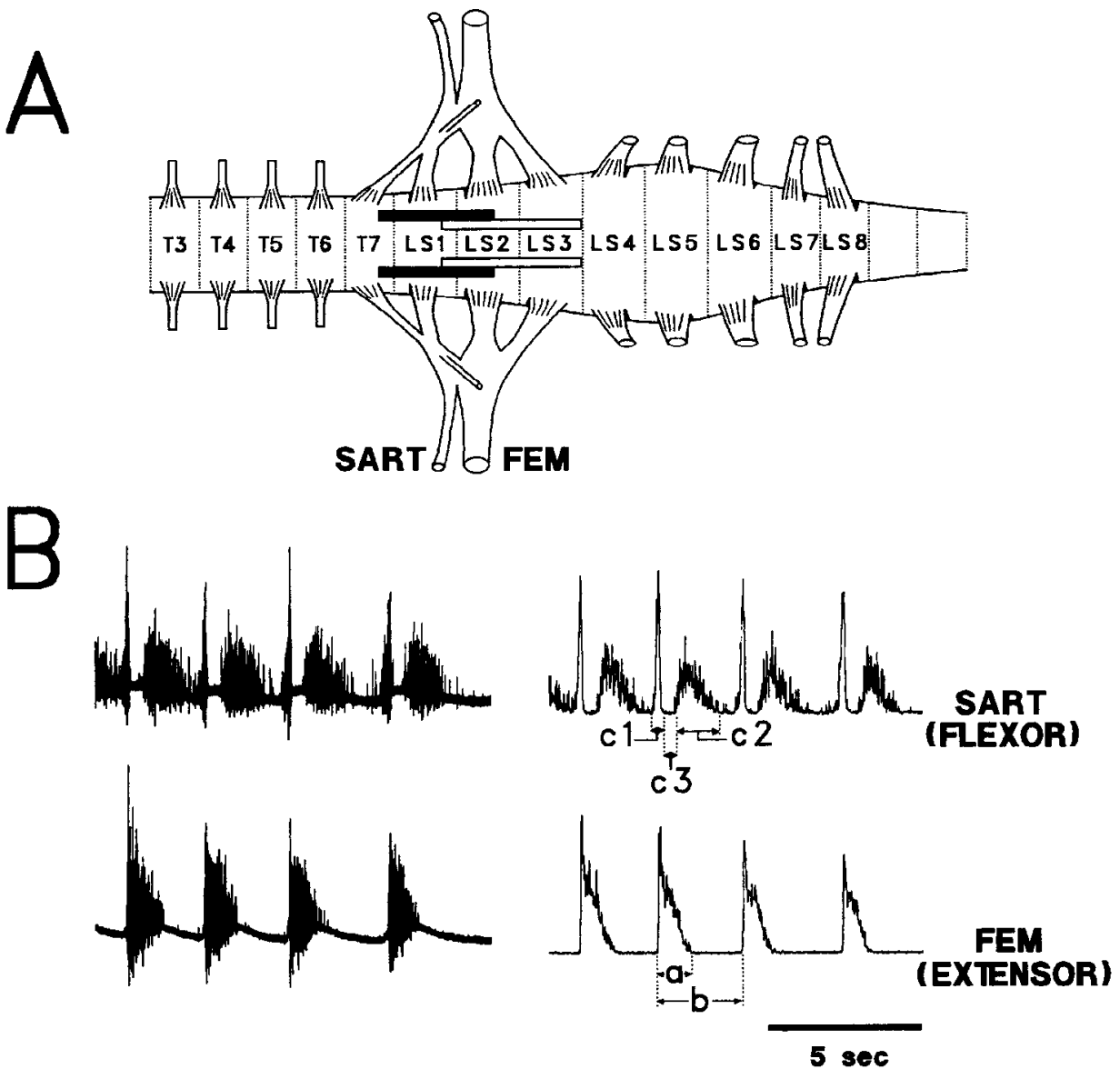

Figure 1. A, Diagram of the lumbosacral spinal cord of the chick embryo, showing the SART and FEM muscle nerves and ventral roots from which we recorded. The hindlimb is innervated by segments $T 7-L S 8$. In most experiments the unlesioned cord also included several thoracic segments (T3-T7). The rostrocaudal extent of the SART (solid bar) and FEM (open bar) motoneuron pools is also shown. $B$, Example of the rhythmic motor output recorded from the $S A R T$ and $F E M$ muscles nerves. Burst parameters were measured on the rectified integrated neurograms (right panel in $B$ ). a, duration of extensor activity; $b$, cycle duration; $c 1$ and $c 2$, duration of the synchronous and second burst of flexor activity, respectively. The end of the $c 2$ period was defined as the time the integrated activity returned to the baseline, disregarding isolated spikes that may sometimes appear. Measurements of $c l$ were only made when the SART cycles occurred in isolation from each other. The inhibitory period in the SART discharge is $c 3$. mogenic networks appear to be localized to the ventral part of the cord. In addition, we have found that the rostrocaudal synchronization of motoneuron activity is mediated, in part, by a propriospinal fiber system running in the ventrolateral white matter. The results also suggest that the neural mechanisms responsible for alternation of flexors and extensors are localized to a circumscribed region, dorsomedial to the lateral motor column (LMC).

Some of this work has appeared in abstract form (Ho and O'Donovan, 1990, 1991) and has been discussed briefly in a review (O'Donovan et al., 1992).

\section{Materials and Methods}

All experiments were performed on the isolated spinal cord of White Leghorn chicken embryos between embryonic day 9 (E9) and E13. The embryo was removed from the egg, decapitated, and eviscerated in an organ bath superfused with Tyrode's solution cooled to about $10^{\circ} \mathrm{C}$. The solution was oxygenated with $95 \% \mathrm{O}_{2}, 5 \% \mathrm{CO}_{2}$ and $\mathrm{pH}$ maintained at 7.3. A dorsal laminectomy was then performed to expose the spinal cord and to free muscle nerves or ventral roots for recording (Fig. 1A). The bath temperature was raised and maintained at $28^{\circ} \mathrm{C}$ for the rest of the experiment. Spontaneous motor activity could be recorded 3-5 $\mathrm{hr}$ after elevating the bath temperature (Landmesser and O'Donovan, 1984). Episodes of motor activity were also triggered by single-pulse stimuli $(10-30 \mu \mathrm{A}, 0.5 \mathrm{msec})$ applied to the dorsal surface of the thoracic cord. When isolated sections of the cord were stimulated, the electrode was placed on one end of the section. We found no dependence of the evoked rhythmic behavior on the placement of the stimulating electrode.

Neurograms were recorded using a tight-fitting polyethylene suction electrode connected to a high-gain DC amplifier (Grass P16). A wide bandwidth (DC to $10 \mathrm{kHz}$ ) was used to record slow electrotonic potentials, which reflect the depolarization of individual motoneurons
(O'Donovan, 1989a), and propagated spike activity. Signals were digitized (Neuro-corder DR-886, Neuro Data Instruments) and recorded on videotape for further analysis. Sometimes neural activity was integrated with a discrete time window set at $5 \mathrm{msec}$. Cycle duration and phase relationships were measured from the integrated spike records using the EGAA software (RC Electronics) and other programs developed in-house. Figure $1 B$ illustrates the definitions of measurements used in this study.

Segments of the cord were isolated using a vibrating tungsten needle. The needle was mounted on a micromanipulator and could be adjusted to cut through any plane of the cord. Vibration frequency was set at $40-60 \mathrm{~Hz}$, and the maximum excursion of the tip was about $3 \mathrm{~mm}$. Lesions were made in the recording chamber so that motor activity could be monitored before and after the lesion. At the end of the experiment the lesioned cords were fixed, cryostat sectioned, and stained with cresyl violet to determine the extent of the lesions. Lesions in the sagittal or horizontal planes were made on isolated cord sections (generally T7-LS3) and were quantified by measuring the vertical height or horizontal width of the remaining gray matter and expressing this as a fraction of the (unlesioned) contralateral measurements. To assess the uniformity of the lesion along the length of the cord, the vertical height or horizontal width of the remaining gray matter was estimated at three successive positions (separated by $200-250 \mu \mathrm{m}$ ) centered near the middle of the isolated section. The maximum variability of the measurement between the three positions averaged $9.26 \% \pm 1.5 \%$ (mean \pm SEM, $n$ $=13$ ) of the measurement of the contralateral gray matter.

In three experiments we used a cobalt gel around the cord to block synaptic transmission locally. This procedure was necessary because the small size of the lumbosacral cord precluded the use of a split bath (Russell and Wallen, 1980; Rovainen, 1985). The gel contained $10 \mathrm{~mm}$ $\mathrm{CoCl}_{2}$ in $15 \%$ gelatin warmed to about $40^{\circ} \mathrm{C}$. It was then applied to the cord after a Vaseline barrier was built to limit the spread of the gel. The blockade of neurotransmission normally took place within $5 \mathrm{~min}$ as evidenced by the disappearance of rhythmic discharge recorded from motoneurons under the gel. 
A

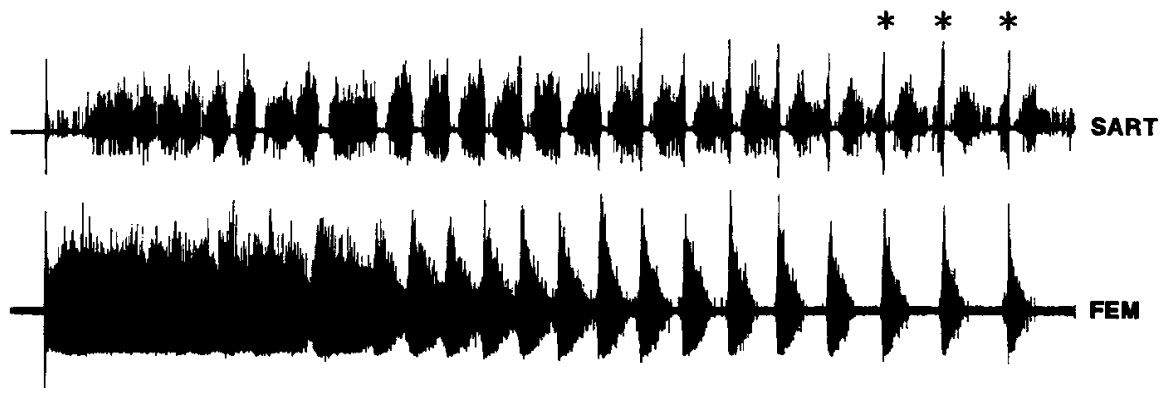

Figure 2. Evoked rhythmic motor activity recorded from the isolated lumbar spinal cord. $A$, An episode of alternating activity recorded from $S A R T$ and FEM muscle nerves after a single stimulus was applied to the rostral cord. The neurograms were high-pass filtered with a cutoff frequency at $100 \mathrm{~Hz}$. The asterisks indicate the synchronous discharge in both muscle nerves. $B$ and $C$ show other patterns of activity that were recorded from more mature preparations (E11-E13). The recordings were made with a wide bandwidth and show discharge superimposed on slow electrotonic potentials. The E13 preparation illustrated in $B$ exhibited two bursts of activity per cycle of depolarization (arrows) following the initial synchronous discharge (asterisks). $C$ and $D$, recorded from an E12 embryo, show brief, high-frequency $(8-10 \mathrm{~Hz})$ synchronous bursts from both flexor and extensor nerves at the beginning of an episode. Calibration: $A-C, 5 \mathrm{sec} ; D, 250 \mathrm{msec}$.
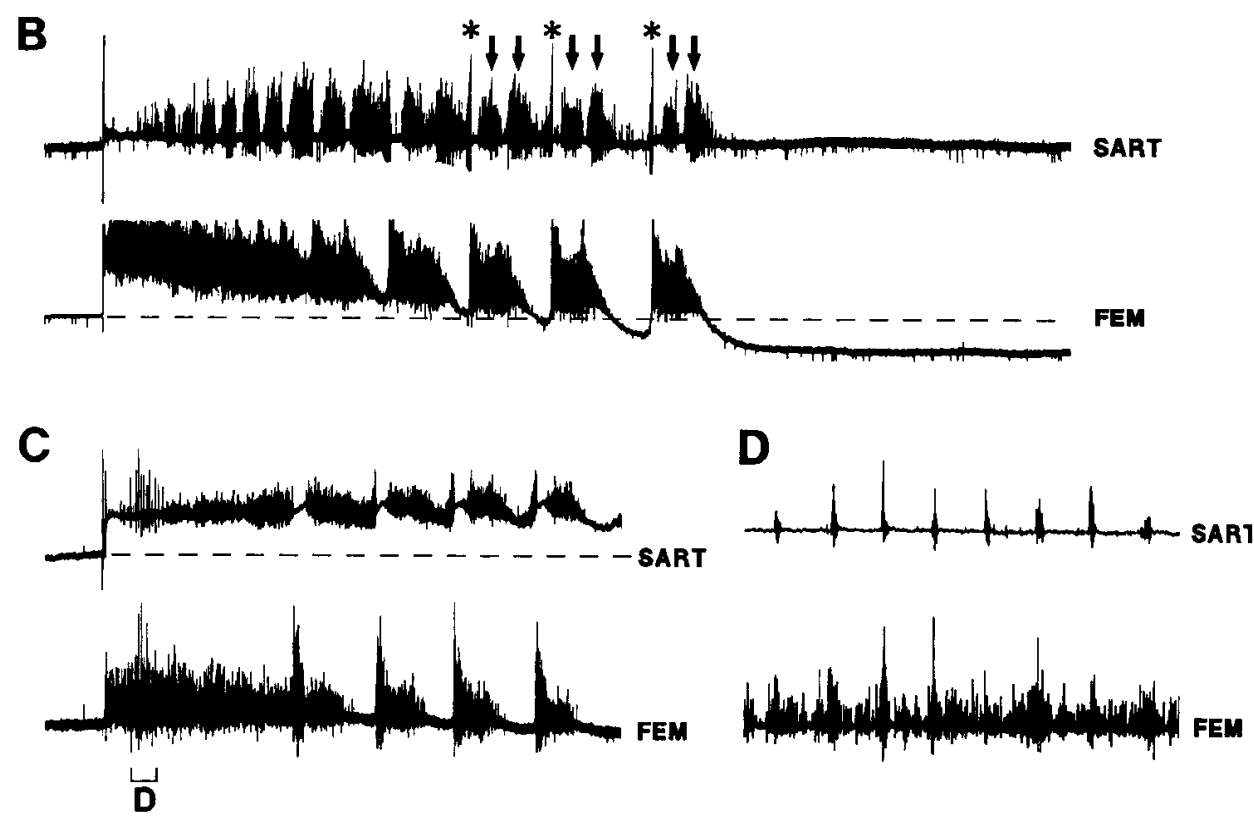

\section{Results}

Normal pattern of motor activity

We have described previously the major features of rhythmic activity produced by the isolated spinal cord and recorded from the ventral roots or muscles nerves (Landmesser and O'Donovan, 1984; O'Donovan and Landmesser, 1987; O'Donovan, 1989a). In such activity a synchronous discharge occurs in both the hip flexor sartorius (SART) and the knee extensor femorotibialis (FEM) nerves at the beginning of each cycle (Fig. $1 B$; asterisks in Fig. $2 A$ ) followed by a silent period in the SART while the FEM continues to fire. The SART discharges again toward the end of the cycle when the FEM activity is diminishing. This alternating pattern becomes more distinguishable toward the later part of an episode.

We also found other forms of motor output particularly in older animals (E12-E14; see Fig. 2B,C). One type of activity consisted of double bursts (arrows in Fig. $2 B$ ) in both FEM and SART following the synchronous discharge (asterisks in Fig. $2 B$ ). Another type of activity consisted of brief, high-frequency $(8-10 \mathrm{~Hz})$ bursts superimposed on the ongoing activity that were synchronized in SART and FEM motoneurons (Fig. 2C,D). This type of activity was seen in $29 \%$ of all E11-E13 preparations used in this study. The brief, synchronized discharges could occur during the silent period of the SART, indicating that they can overcome the potent synaptic inhibition that is known to be present at this time (O'Donovan, 1989a; Sernagor and O'Donovan, 1991). The origin of these discharges is unknown.
Their presence may indicate an altered mode of oscillation of the spinal central pattern generator. Alternatively, they could be derived from synchronized activity in a set of presynaptic inputs that project to both FEM and SART motoneurons. One candidate for such inputs might be the axons of the ventrolateral white matter, which can produce a powerful synchronized excitation of SART and FEM motoneurons (Ho and O'Donovan, 1991).

\section{Coordination of motor activity along the cord}

Each cycle of rhythmic motoneuron activity is known to be synchronized with activity in other lumbosacral segments (Fig. $3 A$; Landmesser and O'Donovan, 1984; O'Donovan and Landmesser, 1987). In the present work we have found that lumbosacral motoneurons are activated synchronously with thoracic motoneurons, indicating that the rhythmic motor output is not restricted to the limb-moving lumbosacral or brachial motoneurons at E9-E11 (Fig. 3B). In cords comprising thoracic and lumbar segments, the activity of lumbar and thoracic segments was well synchronized although discharge in thoracic motoneurons followed that of lumbosacral motoneurons. To illustrate this, we have compared the timing of lumbosacral and thoracic discharge at the beginning of each cycle using either the SART inhibition, which was well defined and abrupt (beginning of period $\mathrm{c} 3$ in Fig. $1 B$ ) or the onset of FEM discharge (beginning of period b in Fig. $1 B$ ) as reference points (Fig. $3 C$ ). The onset of LS7 discharge preceded the onset of SART inhibition by 53 

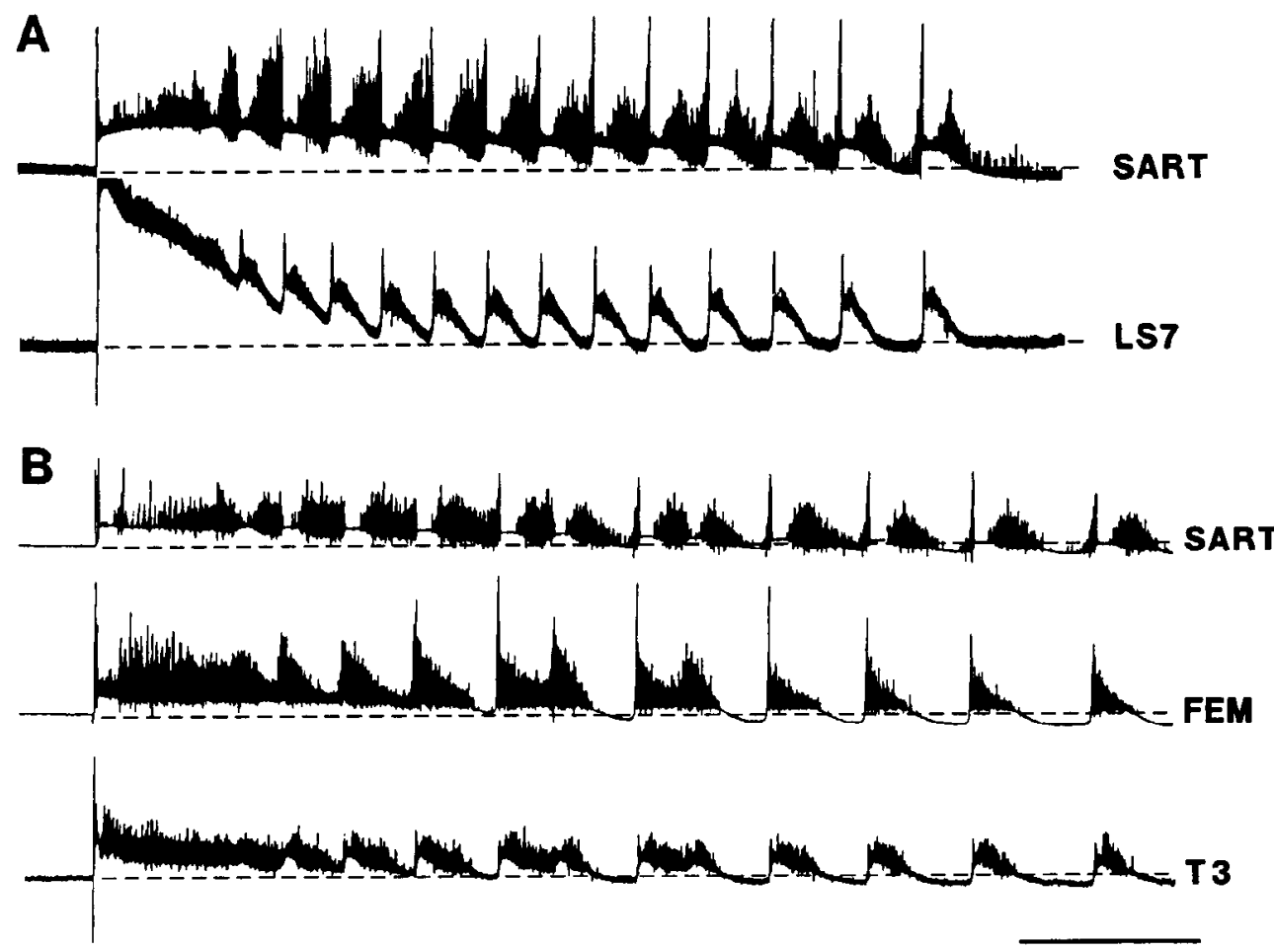

Figure 3. Motor activity is synchronized at different segmental levels. $A$, Motor activity recorded from the $S A R T$ and $L S 7$ of an E 10 preparation during an evoked episode. Tightly synchronized activity occurs in these motor pools, which are several segments apart. $B$, Rhythmic motor activity recorded from a preparation including the tho-

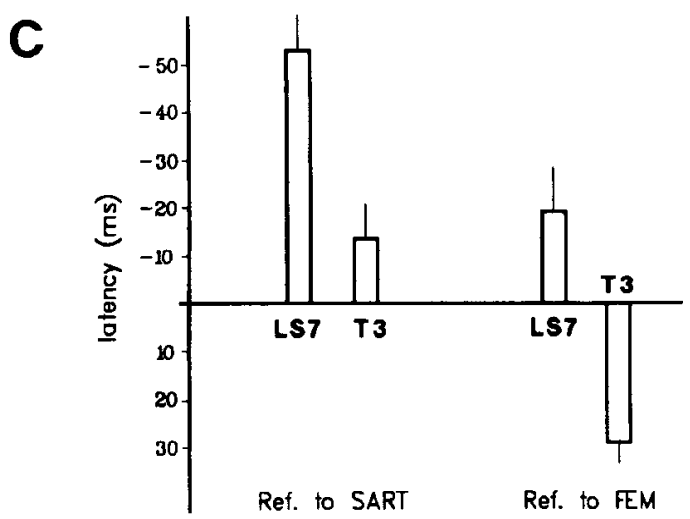
racic and lumbosacral segments (T2LS9) of an E9 cord. Motor activity of the $T 3$ was synchronized with lumbosacral discharges. $C$, Discharge latency of $L S 7$ and $T 3$ referenced to the SART (beginning of the $S A R T$ inhibitory period, which was abrupt and well defined; start of $\mathrm{c} 3$ in Fig. $1 B$ ) or the onset $F E M$ discharge, which was also well defined. A small but significant difference between the onset time of $L S 7$ and $T 3$ motoneurons during each cycle can be observed, $L S 7$ preceding $T 3$ by $40-50$ msec (three preparations, $p<0.001$ ) See text for details. Calibration: $A, 8$ sec; $B, 5$ sec.

$\pm 38 \mathrm{msec}$ (mean $\pm \mathrm{SEM}$ ), whereas $\mathrm{T} 3$ discharge preceded the onset of SART inhibition by $14 \pm 18 \mathrm{msec}(n=109$, three preparations). Thus, the firing of caudal motoneurons (in LS7) occurs approximately $39 \mathrm{msec}$ before the firing of T3 motoneurons. A similar finding was obtained in four other preparations using the onset of FEM discharge as the reference point. In this case the mean onset time of LS7 discharge was $20 \pm 30$ msec (mean + SEM) before the FEM burst, while T3 motoneurons fired $29 \pm 12 \mathrm{msec}$ after the FEM ( $n=93$, four preparations). These differences in latency are small compared to the duration of bursts (1-2 sec; see Fig. 12A) and reveal the near synchrony in the activation of motoneurons at different segmental levels.

\section{Mechanisms underlying the coordination of activity between rostral and caudal segments of the lumbosacral spinal cord}

The location of pathways involved in the synchronization of motor activity between the rostral and caudal lumbosacral segments was investigated in four preparations by selective lesions. We first established that the lateral part of the cord was sufficient to coordinate activity between rostral and caudal segments. Fig- ure 4 illustrates an experiment in which the isolated LS6-LS7 segments were left connected to the rostral cord through a lateral strip of the cord extending from LS4-LS5. Under these conditions rhythmic activity persisted in the caudal segments with the same phase relations as before the lesion. In addition, the number of cycles gencrated per episode was unchanged after the lesion $(6.7 \pm 1.5$ vs $7.6 \pm 2.0$ cycles, mean \pm SEM, before and after the lesion, respectively; three preparations).

This observation suggested that the coordination of rostral and caudal activity was mediated by axons traveling in the ventrolateral white matter tracts (VLT). To establish if these tracts were necessary for such coordination, they were transected. Following such lesions $(n=3)$ the amplitude and duration of each cycle of activity produced by the caudal segments were reduced (compare iLS7 in Fig. $5 A, B$ ) and the firing of caudal motoneurons was significantly delayed in each cycle. In controls, the ipsilateral LS7 (iLS7) and contralateral LS7 (cLS7) motoneurons fire synchronously in each cycle (Fig. $5 A$ ). The onset of ipsilateral discharge referenced to the contralateral side was $-2.0 \pm 4.9 \mathrm{msec}$ (mean $\pm \mathrm{SE}, n=56$ cycles, three preparations). Following ipsilateral VLT lesions between LS4 and 


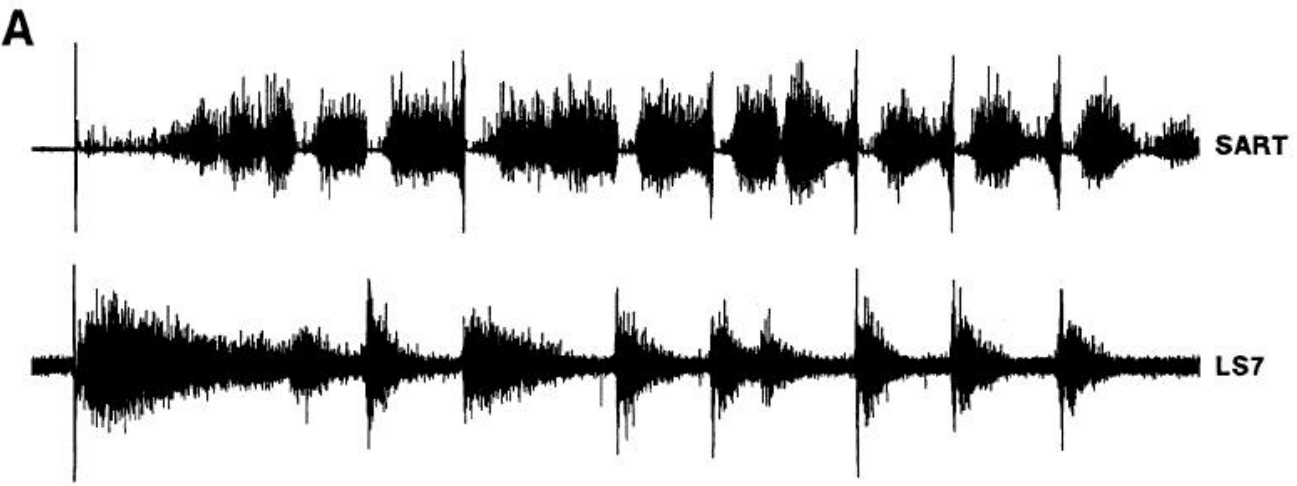

Figure 4. The lateral part of the cord B

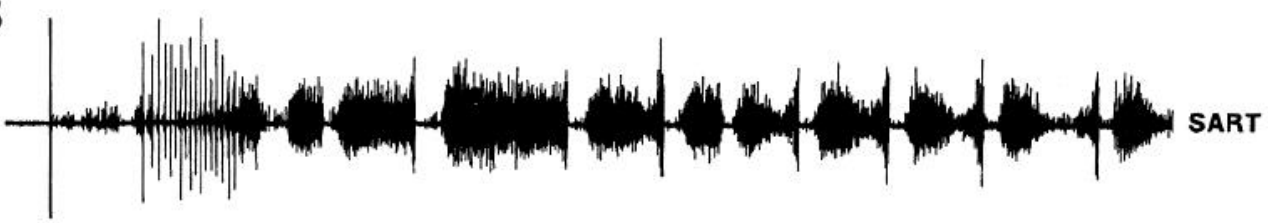
is sufficient to maintain synchronized rostral and caudal ventral root activity. $A$, Control recordings from the $S A R T$ and $L S 7$ segmental nerves of a T6-LS7 cord piece in an $\mathrm{E} 10$ preparation. $B, \mathrm{~A}$ lesion was made to remove most of the medial gray matter and the contralateral cord between LS4 and LS6. Motor activity in $L S 7$ remained synchronized with the rostral activity during an evoked episode. $C$, Photomicrograph of a piece of cord through the remaining lateral strip at the level indicated by $C$ in the drawing. $D$, Photomicrograph of a region of unlesioned cord (position $D$ in the drawing) to illustrate mediolateral extent of the remaining lateral strip. Calibration, $A$ and $B, 5 \mathrm{sec}$. Scale bar, $C$ and $D, 500 \mu \mathrm{m}$.
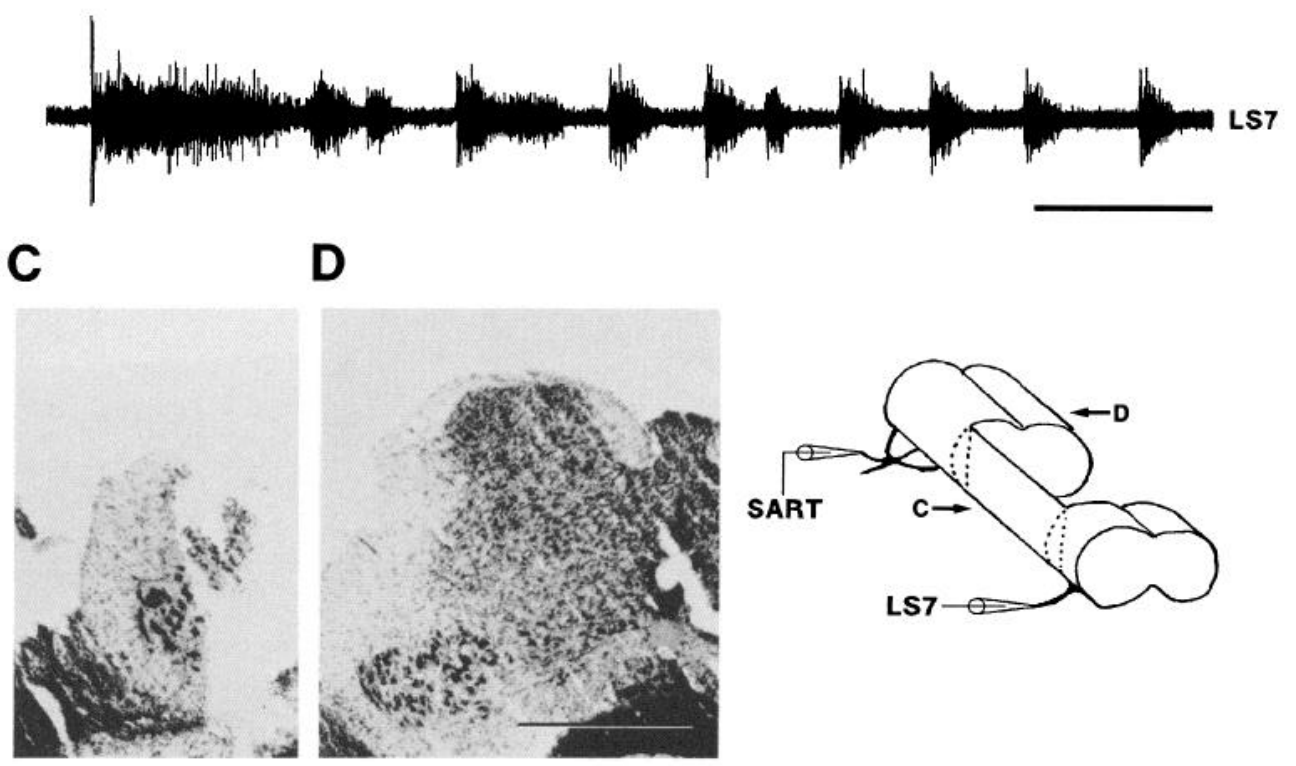

LS7 (Fig. $5 E$ ), the LS7 activity on the lesioned side was delayed by $189 \pm 8.2 \mathrm{msec}$ ( $n=93$ cycles, three preparations) with respect to the unlesioned contralateral side (see Fig. $5 F$ ). The delay could result from an alteration in the coupling between rostral and caudal segments, or it could simply be due to the reduced synaptic drive experienced by caudal motoneurons after the tract lesion.

Following the lesion, the iLS7 ventral root potentials were reduced in amplitude and had an altered shape. In the unlesioned cord the potentials tended to plateau in each cycle, whereas in the lesioned animals they decayed rapidly after an initial peak. These residual potentials could have originated from synaptic activation through the remaining gray matter or from transmission through the uncut, dorsal pathways. In three experiments we investigated this more directly by blocking synaptic transmission between the rostral and caudal segments with a cobalt gel applied over the lesion site (Fig. $5 D$; also see Materials and Methods). This procedure resulted in abolition of caudal motor discharge and a further reduction in the amplitude of synaptic potentials recorded from the LS7 ventral root on the lesioned but not on the unlesioned side. These results suggest that residual activity in caudal motoneurons following a VLT lesion is largely mediated through the gray matter. The small motoneuron potentials remaining after the lesion and the $\mathrm{Co}^{2+}$ gel may be due to weak coupling in the uncut, dorsal white matter tracts.

The minimal effect of the $\mathrm{Co}^{2+}$ gel on the contralateral LS7 motoneurons (Fig. $5 \mathrm{C}$ ) allows us to draw two conclusions. First, that axonal fiber tracts traveling through the gel are adequate to maintain the coordinated activity of rostral and caudal motoneurons in the absence of synaptic transmission in the intervening gray matter. This result confirmed the lesion experiments (Fig. 4) showing that coordinated rostral and caudal activity was preserved providing that the lateral tract axons were left intact. Second, we can conclude that the $\mathrm{Co}^{2+}$ is acting locally, within or near the gel, so the depression of the LS7 activity on the lesioned side is not due to leakage of $\mathrm{Co}^{2+}$ from the gel.

Collectively, these experiments indicate that the tight synchrony normally exhibited by lumbosacral motoneurons is mediated primarily by axons traveling in the lateral and ventral 


\section{A. CONTROL}
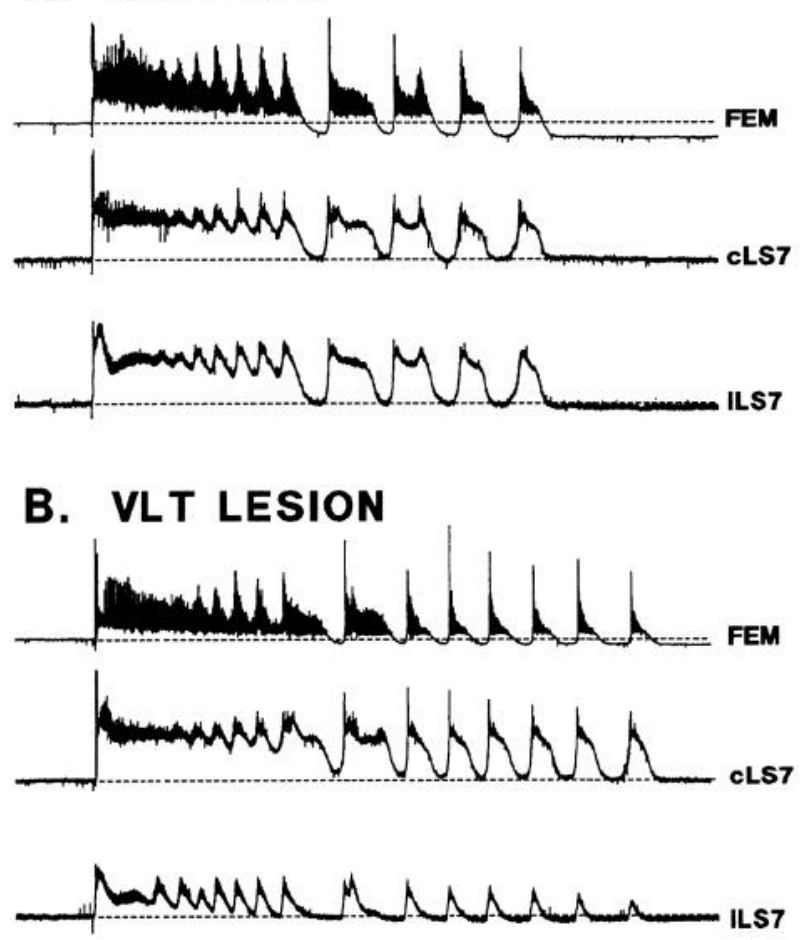

ILS7
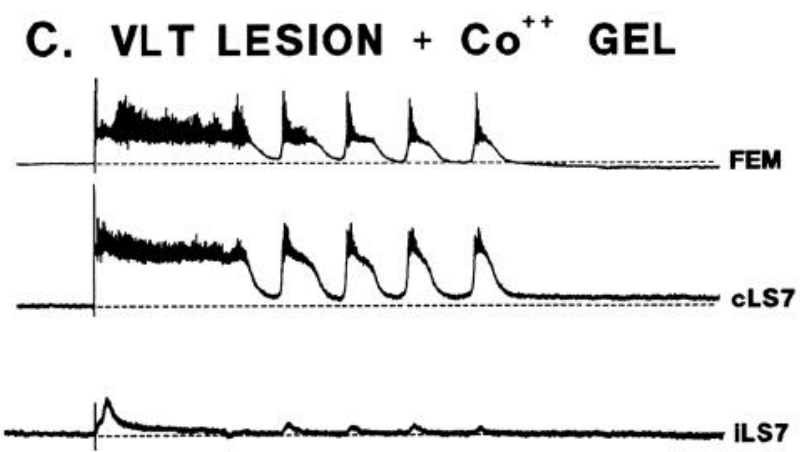

n

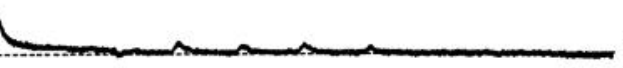

ILS7

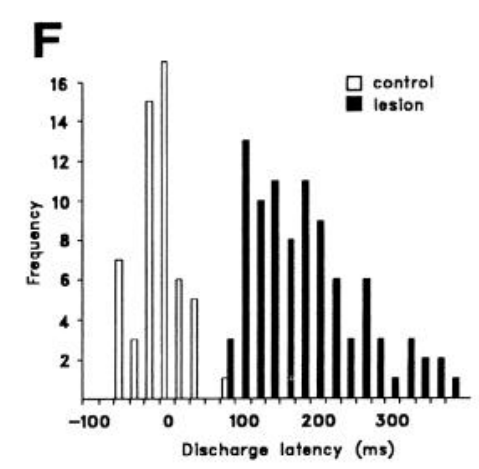

Figure 5. The effects of a ventrolateral tract lesion on the coordination of motor activity at different segmental levels. $A$, Neurograms recorded from the FEM, contralateral LS7 ( $c L S 7)$, and ipsilateral LS7 ( $i L S 7)$ segmental nerve of an E11 preparation consisting of segments T6-LS9. The cord was hemisected caudal to LS1 so that motor pools in LS7 could be driven by activity in the ipsilateral rostral segments, but not by contralateral activity. $B, i L S 7$ ventral root potentials were reduced and discharge was delayed after cutting the ipsilateral ventrolateral tracts between LS4 and LS6. $C$, A cobalt gel $(10 \mathrm{~mm}$ $\mathrm{CoCl}_{2}$ in $15 \%$ gelatin) was added over the lesion site and the corresponding contralateral cord to block synaptic transmission in the remaining gray matter. This further reduced the activity of $i L S 7$ during evoked rhythmic activity. $D$, Schematic illustration showing the preparation, the arrangement of recording electrodes and the location of the $\mathrm{Co}^{2+}$ gel. $E$, Camera lucida drawing of the cord section at the level of the ipsilateral ventrolateral tract lesion (labeled $E$ in $D$ ). The shading indicates the extent of the lesion. $F$, Histogram showing the change in discharge latency of the ipsilateral LS7, referenced to the contralateral LS7, in control and lateral tract-lesioned preparations $(n=3)$. The mean discharge latency was significantly delayed after the lesion ( $p<$ $0.001)$. white matter tracts and that the synaptic drive to caudal motoneurons depends on these tracts and on synaptic pathways through the gray matter.

\section{Regionalization of rhythm-generating networks within the rostrocaudal axis of the lumbosacral cord}

In the previous section we showed that caudal activity remains synchronized to rostral activity provided the lateral tracts are intact and that discharge of caudal motoneurons is abolished and caudal root potentials are greatly depressed when the ventral and lateral tracts are sectioned and synaptic transmission is blocked in the remaining gray matter. These findings raise the possibility that the caudal activity depends in some way on activity originating rostrally and, furthermore, that the rostral lumbosacral segments might be more capable (than the caudal segments) of generating rhythmic activity. We investigated this idea by separating the cord into pieces of varying length and determining the ability of each piece to generate rhythmic activity. We found that all such pieces (typically four to five seg- ments long) including thoracic (T2-T6; $n=3$ ), rostral lumbosacral (T7-LS3; $n=5$ ), and caudal lumbosacral (LS4-LS9; $n=$ 5 ) could produce rhythmic activity (Fig. 6). This observation confirms earlier studies showing that cord pieces isolated in ovo by cervical or thoracic lesions can produce rhythmic motor output (Hamburger and Balaban, 1963; Oppenheim, 1975) and similar studies in the lamprey (Cohen 1987a,b). We also determined that rhythmic activity could be expressed in a single isolated segment from the rostral lumbosacral cord (LS1; $n=$ 3). The isolated LS1 segment typically exhibited two or three cycles in response to a single stimulus to its dorsal surface, and this could be increased to four or five cycles in the presence of $10 \mu \mathrm{M}$ NMDA. However, in an isolated segment the phasing of motoneuron activity was abnormal (compare Fig. $6 C$ to Figs. $1 B, 2 A)$. We did not investigate the capacity of other isolated, single segments to sustain the rhythm. Together, these findings indicate that the rhythm-generating circuitry is distributed along the spinal cord and is not restricted to a particular region. In this respect the embryonic chick cord appears to be organized 
A
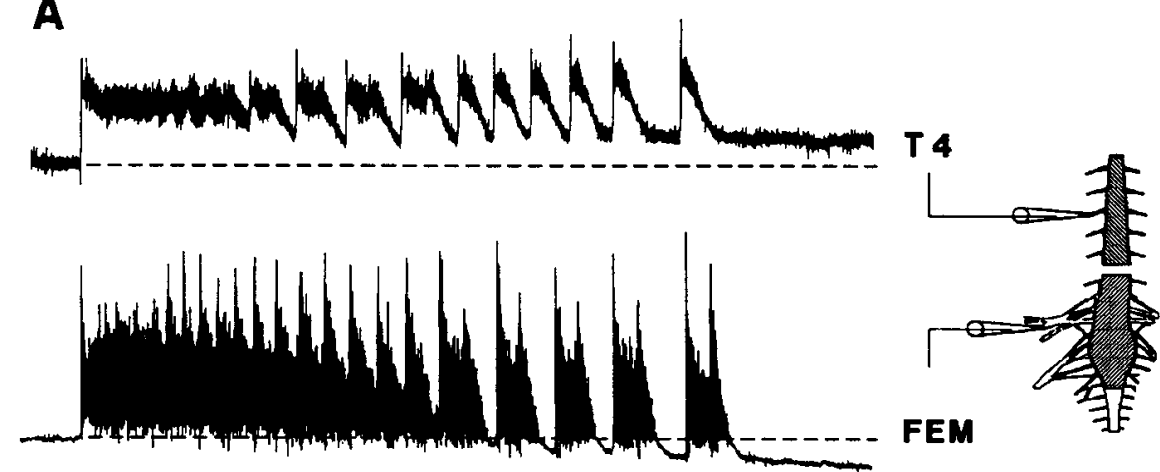

Figure 6. Rhythmic activity is expressed in isolated cord pieces. $A$, Rhythmic motor activity recorded from $T 4$ and FEM after isolating the cord into thoracic (T2-T6) and lumbosacral (T7-LS9) pieces. Both parts were capable of generating evoked or spontaneous episodes of activity. In $B$, the lumbosacral cord was divided into two parts, T7-LS3 and L4-LS9. Recordings were made from FEM muscle nerve and $L S 7$. C, Rhythmic activity can also be recorded from the $S A R T$ muscle nerve in a single segment (LS1) of the lumbosacral cord, although the pattern of activity differed from intact preparations (compare SART activity in Figs. 2, 3). The activity was initiated by a single stimulus to the dorsal surface of the segment and the bath Tyrode solution contained $10 \mu \mathrm{M}$ of NMDA. Calibration: $A$
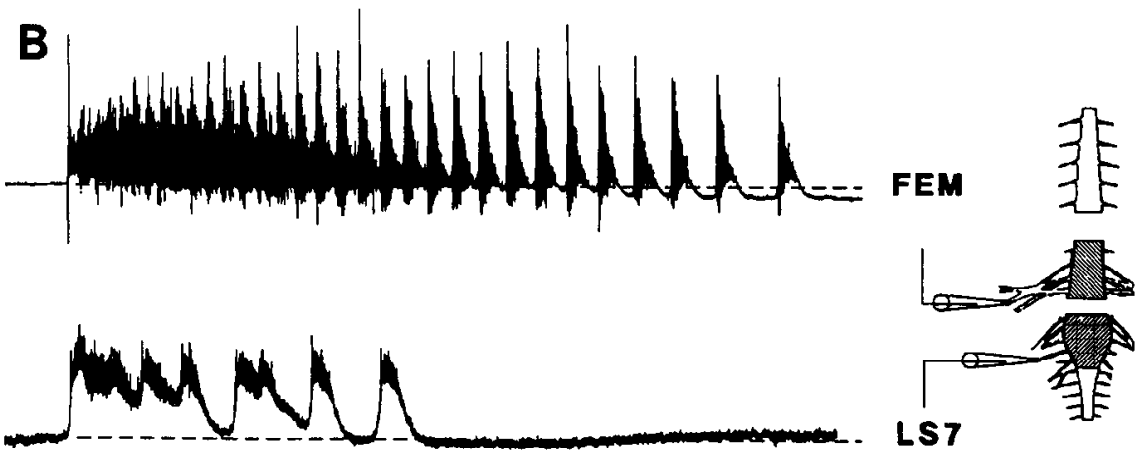

and $B, 10 \mathrm{sec} ; C, 5 \mathrm{sec}$.

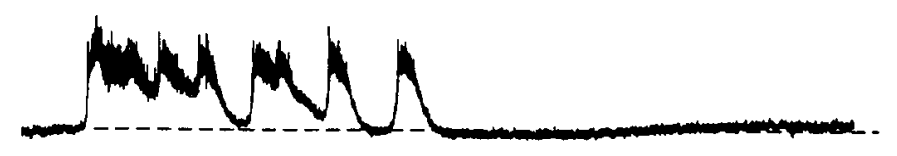

LST
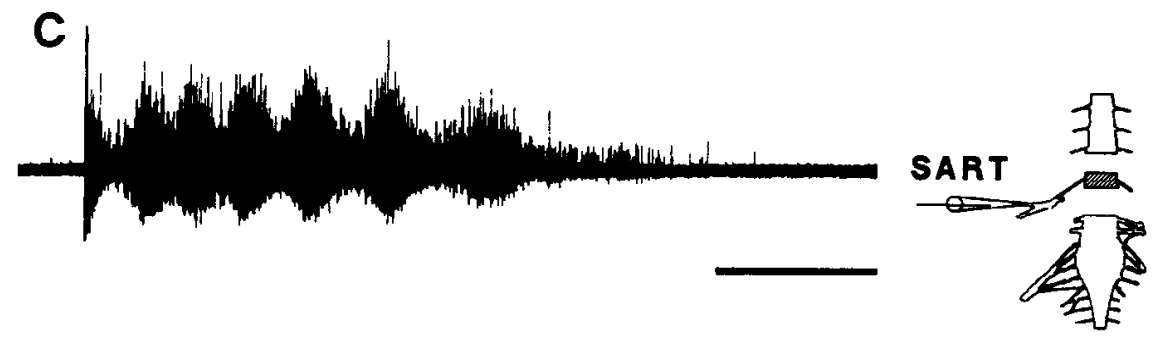

similarly to the lamprey, Xenopus embryo, and turtle spinal cord in which segmental oscillators are coupled along the rostrocaudal axis and are capable of rhythmic activity when isolated in pieces one to two segments long (Cohen and Wallen, 1980; Kahn and Roberts, 1982; Grillner and Wallen, 1984; Mortin and Stein, 1989).

Although rhythmic activity persisted in short pieces of the lumbosacral cord, we did find differences between the rostral and caudal regions. The number of cycles recorded from the caudal cord (LS4-LS9) was only $28 \% \pm 9 \%$ (mean \pm SE, five preparations) of that recorded from the rostral cord (T7-LS3), even though the caudal part contained more segments (see Fig. $6 B$ ). This difference was examined in more detail by successively ablating either the rostral or caudal segments, one at a time, while recording activity from one of the remaining segments. Because of variability in the number of cycles between animals and across developmental stages, the values were normalized to the number of cycles produced by the intact cord before the lesion. When the recording was made from the caudal LS7 ventral root in E11-E12 embryos, the number of cycles per episode dropped immediately after removal of one or two thoracic segments (Fig. 7B). The number of cycles then stabilized at ap- proximately $60 \%$ of control (prelesion) values until the LS2 segment was removed, whereupon there was a rapid decline to about $30 \%$ of control values.

These experiments revealed that the rostral segments are capable of generating more cycles than the caudal segments and are consistent with our hypothesis that activity in the caudal segments is dependent upon activity originating rostrally. The result suggests that the rhythm-generating mechanisms differ in the two regions, although an alternative interpretation is that the rostral cord is more mature than the caudal cord. This alternative suggestion is plausible because the number of cycles generated by the whole cord progressively increases throughout development (O'Donovan and Landmesser, 1987) and a rostrocaudal gradient is known to exist for several aspects of cord development (Hollyday and Hamburger, 1977; Williams et al., 1987). To test this idea, we repeated the experiments in younger embryos (E9-E10) and found that the lesion-induced decline in the number of cycles recorded from the caudal cord was less marked than in the older animals (squares, Fig. $7 B$ ). These developmental changes were investigated more fully by comparing the number of cycles generated by isolated caudal segments (LS3-LS9) at different stages of development. The data 


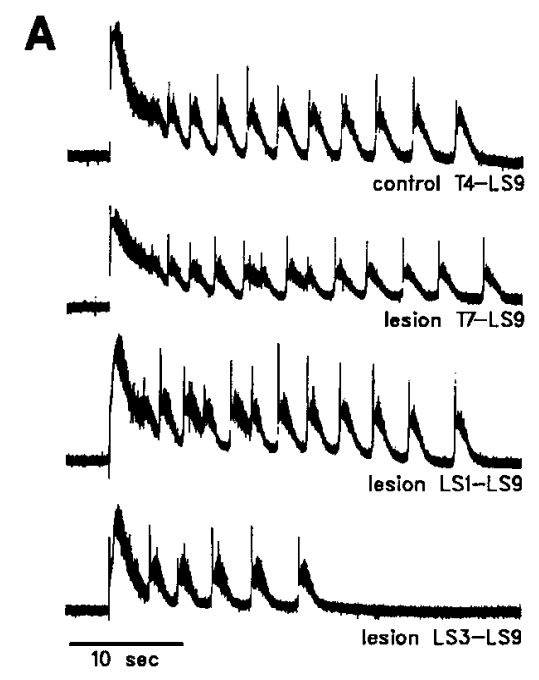

B

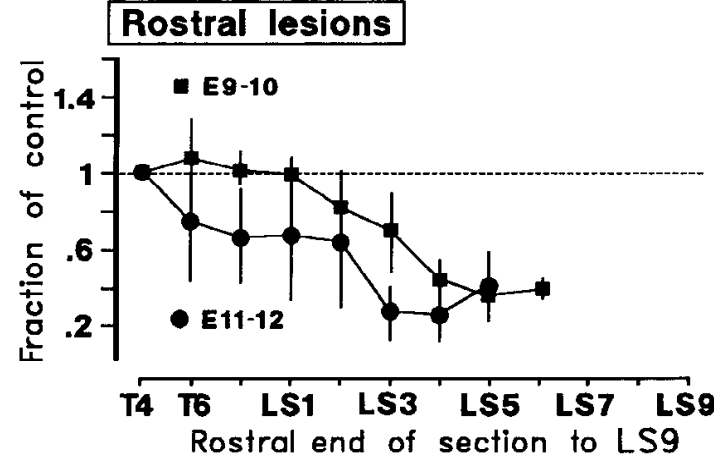

C

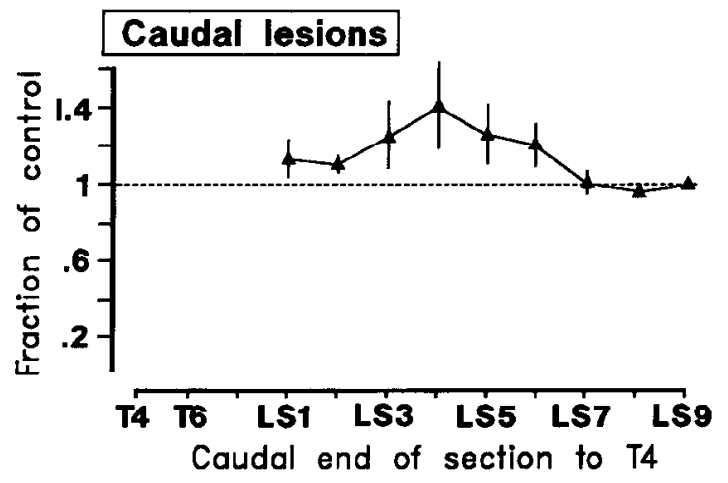

(Fig. $7 D$ ) show a developmental increase in the dependence of caudal activity on rostral activity. At E9, caudal segments produce $80 \%$ of the cycles of the intact lumbosacral cord, whereas by E13 they are only capable of generating $20 \%$ of the cycles. This result suggests that the rostrocaudal specialization becomes more pronounced during development, making it unlikely that the differing capacity of the rostral and caudal lumbosacral cord to generate rhythmic output at E13 is simply due to immature caudal segments.

It might be argued that the depression of the caudal activity is a consequence of the rostrocaudal progression of the lesions, and is therefore an artifact of the lesioning procedure. To investigate this we reversed the direction of the lesions and made them caudorostrally while recording from a rostral root or muscle nerve (Fig. $7 C$, triangles). In this case, progressive caudal lesions resulted in an increase in the number of cycles recorded
Figure 7. The rostral and caudal parts of the lumbosacral cord differ in their ability to generate rhythmic activity. $A$, Recordings from the LS7 ventral root in an $\mathrm{E} 10$ preparation after sequential lesions of rostral cord segments. The length of the piece is indicated to the right of each neurogram. Episodes were evoked regularly, once every $10 \mathrm{~min}$, and data were not taken until about 20 min after each lesion. $B$, Graph of the change in the number of cycles generated in each episode following progressive rostrocaudal lesions (indicated to the right of the graph). The number of cycles per evoked episode was expressed as a fraction of the control (prelesion) value that was obtained from episodes produced by a cord comprising T4-LS9 segments. The data in the graph (mean \pm SD) were pooled from several experiments at two different ages: E9-E10 ( $n=6$, squares $)$ and E11E12 ( $n=6$, circles). $C$, Graph of the change in the number of cycles generated in each episode following progressive caudorostral lesions as indicated to the right of the graph. The data are displayed in the same way as in $B$ and were pooled from four embryos aged E10 E12. $D$, Histogram summarizing the changes in the number of cycles exhibited by isolated caudal segments (LS3LS9) at different stages of development. The values were normalized to the number of cycles produced by the intact cord before the lesion and are expressed as mean $\pm \mathrm{SD}$, averaged from three embryos at each age. from the rostral segments. Removal of the caudal segments from LS5-LS9 resulted in a $40 \%$ increase in the number of cycles recorded from the SART muscle nerve (motoneurons predominantly in LS1). This result indicates that damage is unlikely to account for the reduction in the caudal activity during rostrocaudal lesions. The increased number of cycles following deletion of the caudal segments raises the possibility that these regions normally inhibit or modulate the activity of rostral segments.

\section{Localization of rhythm-generating and pattern-generating networks}

General description of lesions. We performed several experiments to localize the rhythm-generating circuitry in the dorsoventral and mediolateral planes of the cord. For this purpose we employed lesions in the sagittal (mediolateral) and horizontal (dorsoventral) planes to isolate pieces of the spinal cord. We 


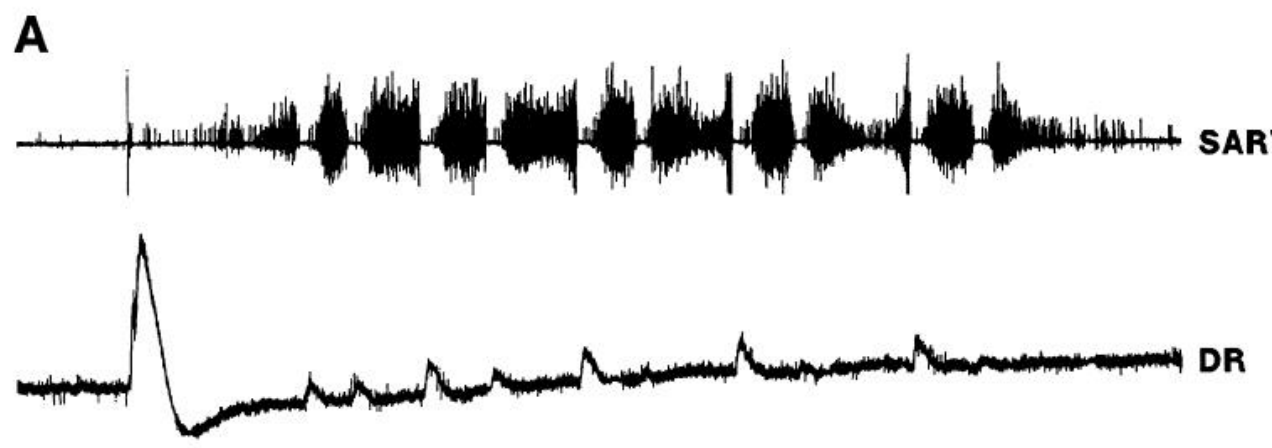

B

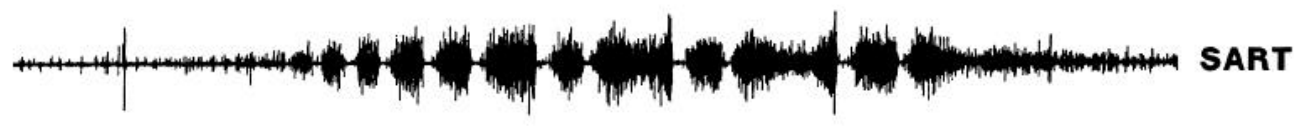

Figure 8. Rhythmic activity is produced by an isolated strip of ventral but not dorsal spinal cord. $A$, Recording from the LS1 dorsal root $(D R)$ and $S A R T$ muscle nerve of an E10, T6-LS4 isolated cord piece. The $S A R T$ neurogram was high-pass filtered. Small rhythmic dorsal root potentials are superimposed on a longer negative potential. $B$, Separation of the cord into dorsal and ventral parts results in abolition of the dorsal but not the ventral rhythmic activity. The recordings were made in the presence of $10 \mu \mathrm{M}$ NMDA. $C$, Photomicrograph showing the level of the separation. The drawing shows the arrangement of recording electrodes. Calibration, $A$ and $B, 5$ sec. Scale bar, $C, 500 \mu \mathrm{m}$.
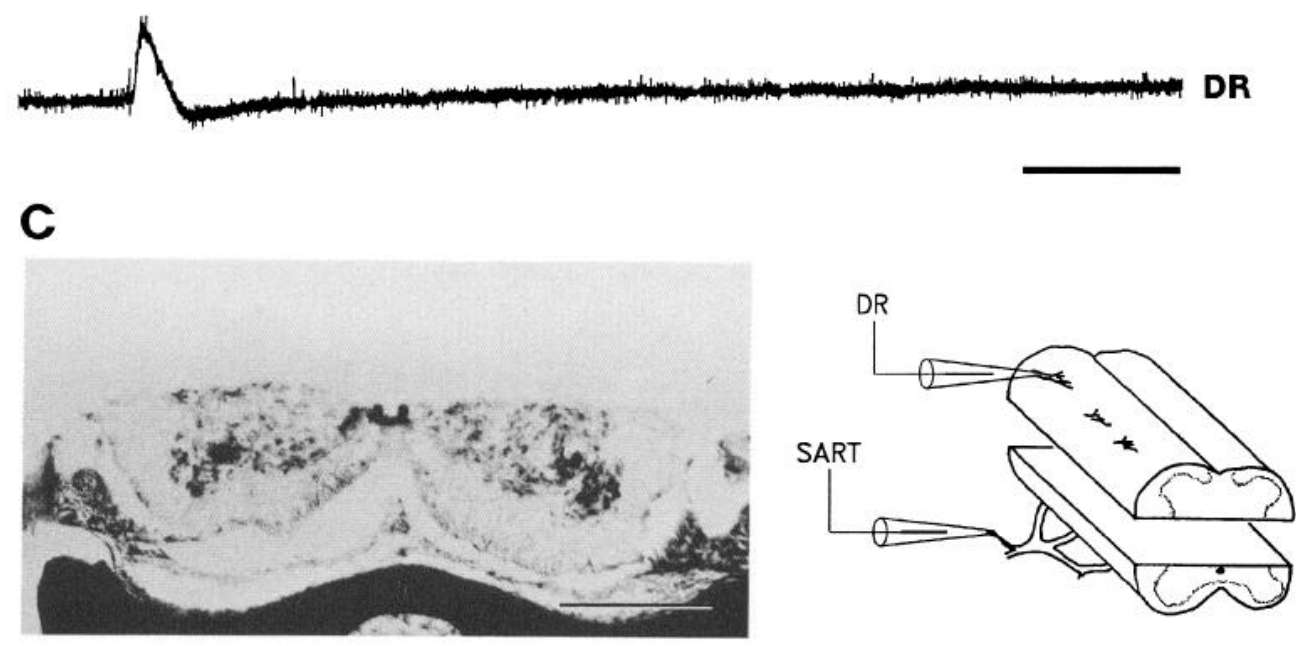

have considered the results separately depending on the effect of the lesions. First, we discuss moderate lesions that produced minimal effects on the motor output, either on the timing of individual cycles or on the phasing of activity in SART and FEM muscle nerves. Moderate lesions included horizontal (dorsoventral) sections down to, but not beyond, the central canal $(n=4)$, and sagittal (mediolateral) sections of $<50 \%$ of the width of one side of the spinal cord $(n=2)$. For moderate lesions the average width (or height) of the remaining gray matter (as a fraction of the contralateral unlesioned side) was $58.5 \%$ (range, $41.8-67.5 \% ; n=6$ ). In two experiments the horizontal and sagittal lesions were combined to isolate the ventrolateral quadrant of the cord. Second, we consider severe lesions. Severe sagittal (medial, $n=4$ ) lesions removed $>50 \%$ of the medial part of the cord up to the medial boundary of the LMC, whereas horizontal (dorsal; $n=3$ ) lesions ablated the dorsal part of the cord to below the level of the central canal. All lesions, moderate and severe, were made over the complete extent of an isolated portion of the rostral cord comprising segments T7-LS3. For the severe medial lesions, the average amount of remaining gray matter was $42.0 \%$ (range, $35.9-48.9 \% ; n=4$ ) of the contralateral side. The remaining gray matter following severe dorsal lesions averaged $23.1 \%$ (range, $10.0-36.3 \% ; n=3$ ).

Moderate lesions with minimal effect on normal activity. Ear- lier studies had suggested that the ventral part of the cord might be specialized to generate rhythmic activity (Hamburger et al., 1966; Provine et al., 1970; Provine, 1971). To test this idea, we separated the cord into isolated dorsal and ventral strips over several segments. Activity in the ventral portion was monitored from the SART motor nerve, while activity in the dorsal part was established by recording dorsal root potentials. In the intact cord rhythmic potentials can be recorded from the dorsal roots during motor activity (Fig. $8 \mathrm{~A}$ ), as is the case in other animals (see Baev, 1978; Dubuc et al., 1985). In the isolated chick cord the potentials are smaller and briefer than the corresponding ventral root potentials and follow an initial large depolarization at the onset of the episode (Fig. 8A). After dividing the cord into dorsal and ventral halves $(n=4)$, the phasic dorsal root potentials could no longer be evoked even in the presence of bath-applied NMDA (10 $\mu \mathrm{M} ; n=2)$. By contrast, rhythmic motor activity persisted in the ventral part of the cord (Fig. $8 B$ ) with normal phasing between FEM and SART motoneurons (not shown). This finding indicates that ventral circuitry alone is sufficient to generate the normal pattern of rhythmic activity (see also Fig. 9) and suggests that the dorsal root potentials are generated by synaptically driven activity in dorsally located interneurons.

In a second set of experiments $(n=2)$, we examined the 

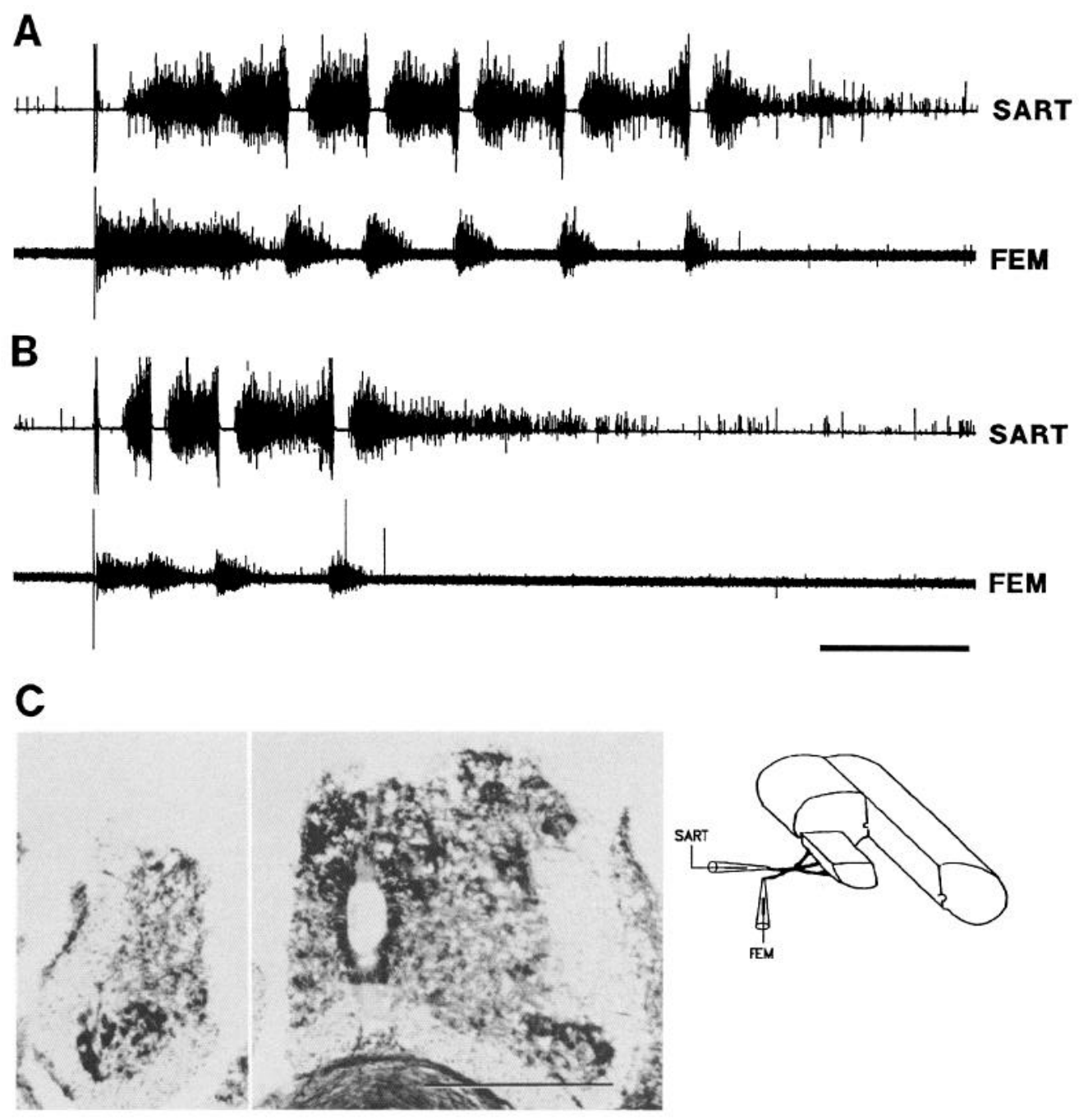

Figure 9. Neuronal elements in the ventrolateral cord are sufficient to generate patterned rhythmic activity. $A$, Recordings from the $S A R T$ and $F E M$ nerves of an E9, T7-LS3 cord piece before lesioning. $B$, Patterned rhythmic activity persists after removing the medial and dorsal parts of the cord over the entire extent of the isolated piece. The alternating pattern between flexor and extensor motoneurons remains, although the number of cycles is decreased. $C$, Photomicrograph of a transverse section of the isolated ventrolateral quadrant (left) and the remaining part of the cord, including the contralateral side, at a more rostral level (right). The drawing illustrates the lesion and position of recording electrodes. Calibration, $A$ and $B, 5$ sec. Scale bar, $C, 400$ $\mu \mathrm{m}$.

dependence of patterned motor activity on neurons located in the medial part of the cord. For this purpose we monitored the pattern of SART and FEM activity following removal of selected portions of the medial cord over several segments (lesions illustrated in Fig. 12). We found that deletion of about one-half of the medial cord produced little change in the alternating pattern of activity between these antagonists although more pronounced medial lesions did affect the pattern (see below). These results indicate that motor activity, with normal antagonist phasing, persists with both dorsal and medial ablations and suggests that the ventrolateral part of the cord might contain the necessary elements for the production of patterned rhythmic activity. To test this idea we combined dorsal and medial lesions to isolate a ventrolateral quadrant of the cord over several segments (T7-LS3). The isolated quadrant was completely detached from rostral and caudal connections to the remaining cord. Rhythmic activity persisted in such reduced preparations $(n=2)$, and the alternation of SART and FEM activity was largely unchanged (Fig. 9B). These findings indicate that the ventrolateral quadrant of the cord contains the essential neuronal elements for the production of patterned motor activity in the isolated chick spinal cord.

Although the phasing and timing of activity were largely unchanged following moderate lesions, we did observe a small
(20\%) but significant reduction in the number of cycles in each episode (six preparations, $p<0.01$ ). This effect may be a nonspecific consequence of removing neural tissue, because a similar reduction (14\%) was observed when the cord was hemisected (seven preparations, $p<0.01$ ).

Severe lesions disrupting normal activity. Following medial lesions up to the medial border of the LMC $(n=4)$ or dorsal lesions below the central canal $(n=3)$, the pattern of activity in SART and FEM motoneurons was altered. Figure 10 illustrates a medial lesion close to the LMC that resulted in abolition of the SART inhibitory period (arrows) after the first one to two cycles and reduced the duration and amplitude of FEM activity. This changed the phasing of activity between SART and FEM from alternation to coactivation. After the lesion, the amplitude and duration of both FEM and SART discharge declined progressively throughout the episode (Fig. 10B). Some recovery of the alternating pattern occurred in the presence of $10 \mu \mathrm{M}$ NMDA (arrowed cycles Fig. $10 \mathrm{C}$ ), although the change from alternation to coactivation still occurred toward the end of the episode as the amplitude and duration of successive bursts decreased. Despite these marked effects on the phasing of activity, there was no change in the number of cycles per evoked episode (six preparations, $p>0.1$ ). A similar result accompanied dorsal lesions in which the gray matter was removed down to, or below, the 
A

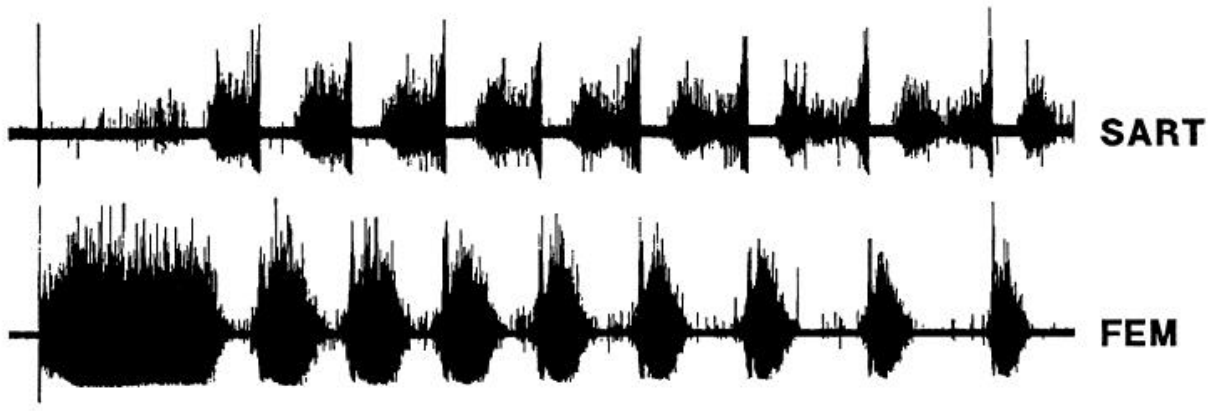

B

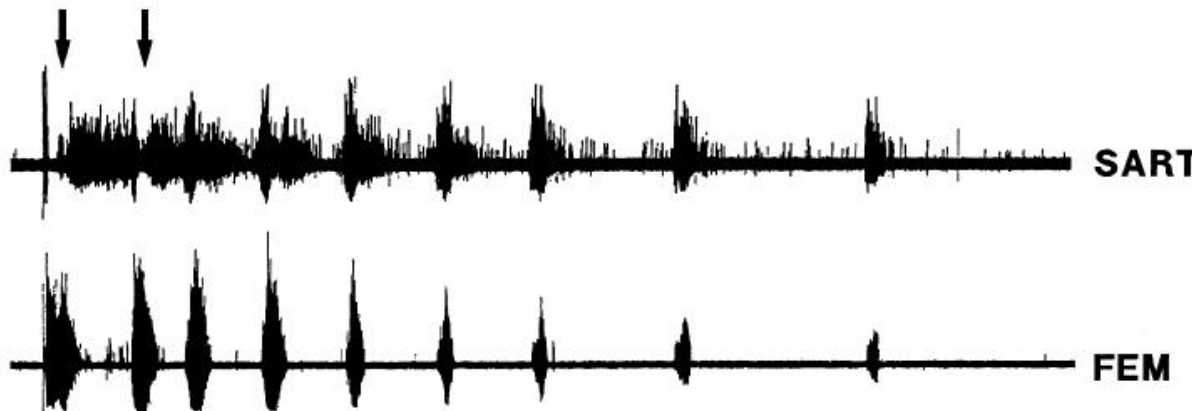

Figure 10. Medial lesions close to the motor column disrupt the alternation of FEM and SART. A, An evoked episode of alternating activity recorded from $S A R T$ and FEM nerves of an E9, T7-LS4 cord piece before lesioning. $B$, A medial lesion of the complete T7LS4 piece of cord reduces the duration and amplitude of the FEM discharge and the duration of the $S A R T$ inhibitory period (arrows) after the first few cycles, resulting in coactivation of the $S A R T$ and FEM. C, The coactivation was partially reversed, in the first few cycles (arrows), by the addition of 10 $\mu \mathrm{M}$ NMDA. $D$, Photomicrograph of a transverse section of the spinal cord to show the extent of the medial lesion. The drawing illustrates the lesion and the position of the recording electrodes. Calibration, $A-C, 10 \mathrm{sec}$. Scale bar, $D$, $400 \mu \mathrm{m}$.

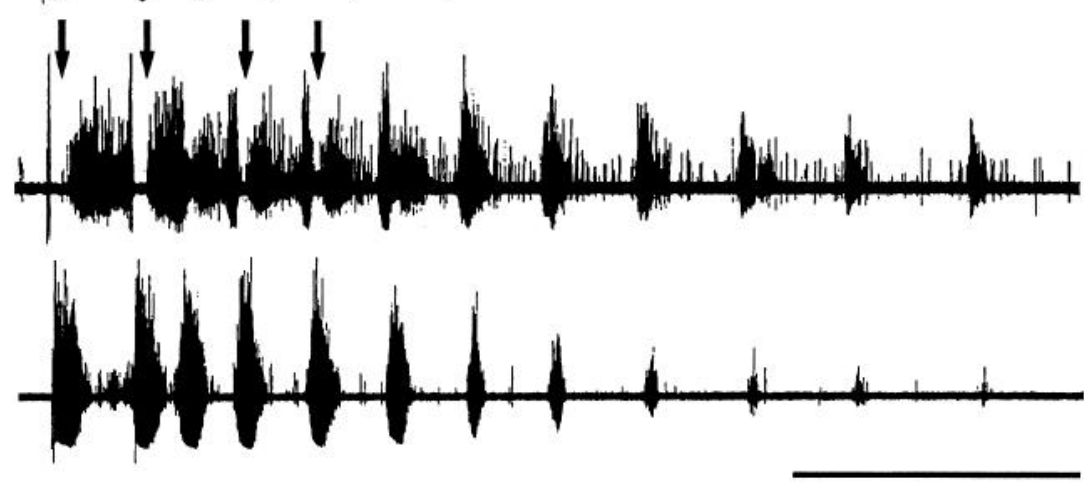

D

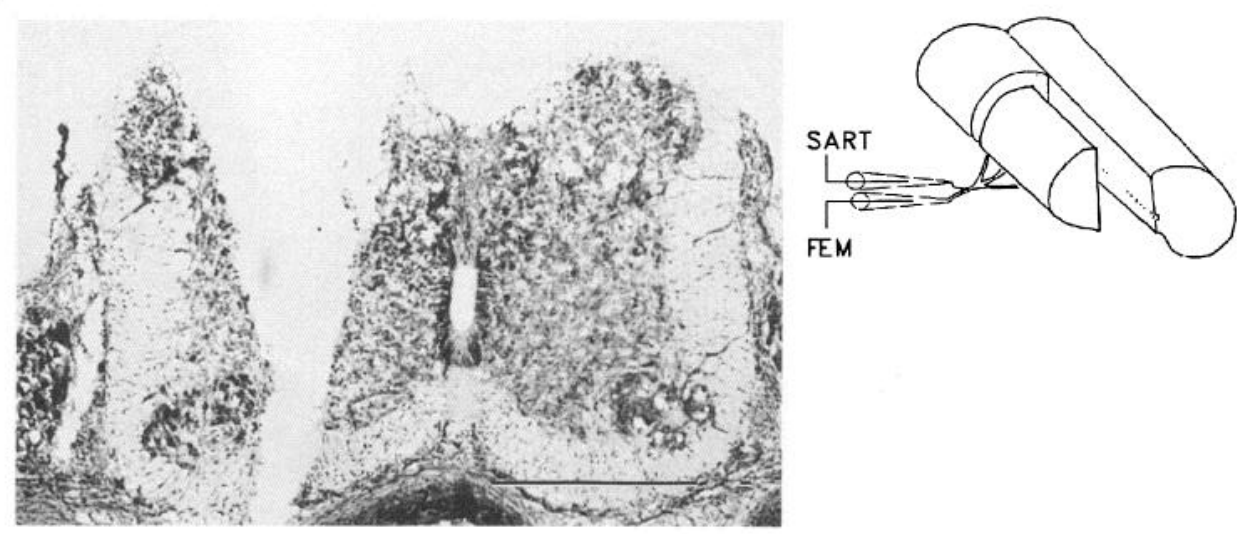

level of the central canal. In such preparations the SART inhibition was reduced (compare SART in Fig. $11 A, B$ ) or abolished (compare SART in Fig. 11C,D) depending on the severity of the lesion. Remarkably, however, despite the removal of the majority of the gray matter, rhythmic motor activity was preserved, and its timing was largely unaffected (see Fig. 12D).

\section{Quantitative description of dorsoventral and mediolateral lesions}

To examine the results of the various lesions quantitatively, we documented their effects on the duration of SART and FEM bursts, the duration of the SART inhibitory period, and the cycle period. These findings are summarized in Figure 12.

Control preparations and moderate lesions. In control episodes the cycle period progressively lengthened after a long initial cycle, which was probably the result of rapidly recurring activity at the onset of the episode (O'Donovan and Landmesser, 1987). The average cycle period was about $2 \mathrm{sec}$ in the second and third cycle, lengthening to approximately $3 \mathrm{sec}$ toward the end of the episode (Fig. 12A). As the episode progressed, the burst duration of the SART increased from $1.3 \mathrm{sec}$ to $1.8 \mathrm{sec}$, while the FEM decreased slightly $(1.5 \mathrm{sec}$ at the second 

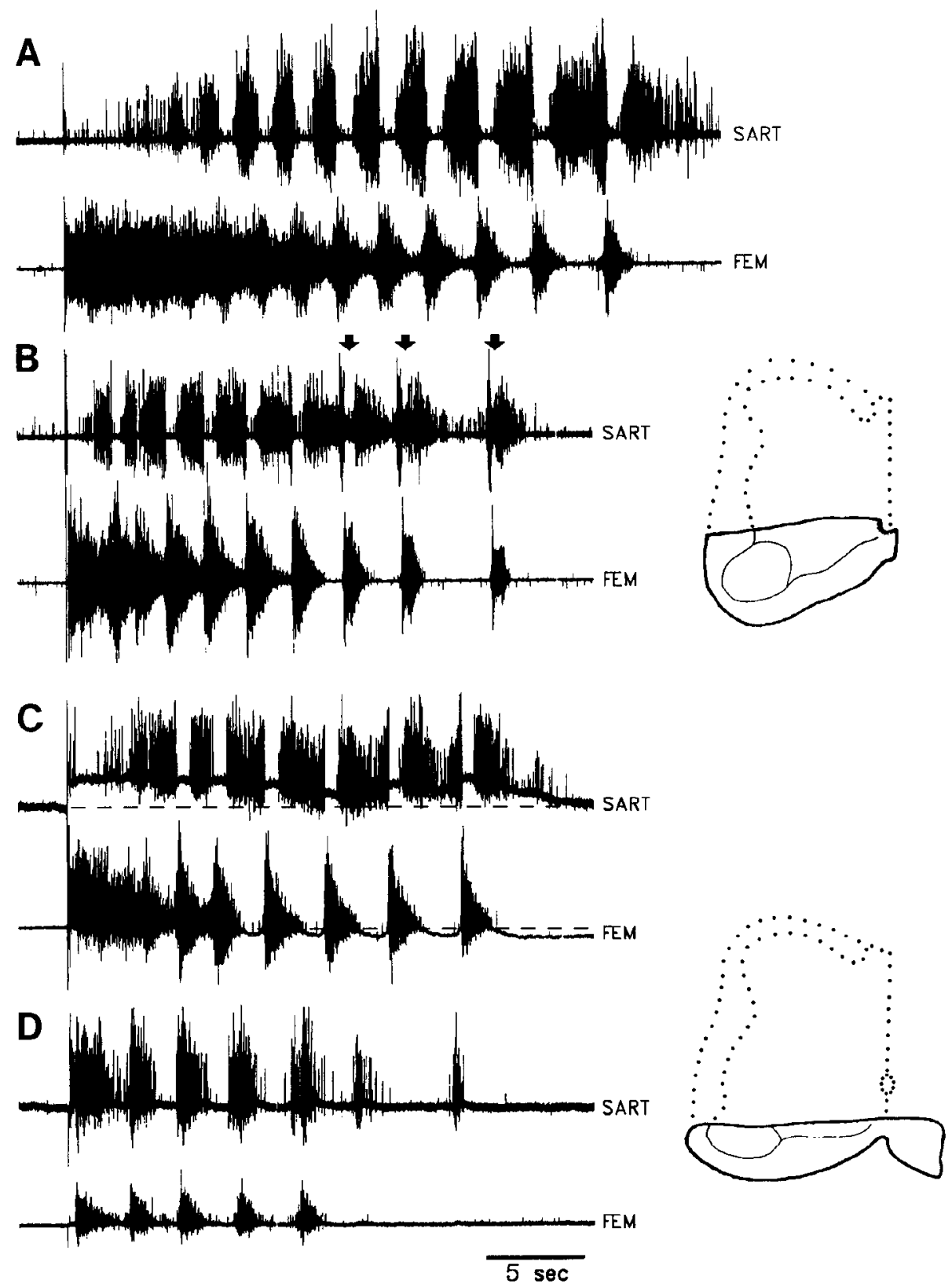

Figure 11. Rhythmic motoneuron activity persists after removal of most of the dorsal part of the spinal cord. $A$, An episode of rhythmic activity from an E10, T7-LS4 cord piece before lesioning. $B$, Following removal of the dorsal part over the entire cord portion (indicated by the diagram to the right of the records), rhythmic activity persisted but the $S A R T$ inhibition was abolished toward the end of the episode (arrows). $C$, Control recording from another $\mathrm{E} 10$, T7-LS4 preparation before lesioning. $D$, Ablation of most of the dorsal spinal cord (indicated to right) resulted in a thin ventral strip that still expressed rhythmic activity. Note that the $S A R T$ inhibitory period was abolished resulting in coactivation of the $S A R T$ and FEM discharge.

cycle to $1.3 \mathrm{sec}$ at the ninth cycle). In contrast, there was little change in the duration of SART inhibition, which remained constant at about $0.6 \mathrm{sec}$ (Fig. $12 \mathrm{~A}$; see also O'Donovan and Landmesser, 1987). These profiles were not altered following moderate dorsal or medial lesions (including two ventrolateral quadrant isolations) except near the end of the episode when the duration of both FEM and SART bursts tended to decrease to about 1.1 and $1.4 \mathrm{sec}$, respectively (Fig. 12B). The cycle duration also decreased and became more variable toward the end of the episode in the moderate lesion group (Fig. 12B). This suggests that neural elements removed by the moderate lesions may contribute to the stability of the cyclic properties of the network but are not themselves essential for the generation of the rhythm or the patterning of activity.

Severe medial and dorsal lesions affect the duration of excitation and inhibition rather than cycle timing. As illustrated in Figure $12, C$ and $D$, severe medial and dorsal lesions predominantly affect the duration of the SART and FEM activity and the SART inhibition. Following severe medial lesions, the SART inhibition was reduced to less than $0.1 \mathrm{sec}$ by the fifth cycle and the FEM burst duration was reduced by about $50 \%$, from 1.5 $\mathrm{sec}$ in control to $0.8 \mathrm{sec}$ after the lesion. After severe dorsal lesions, the SART inhibition was reduced to about $0.25 \mathrm{sec}$ and the FEM burst duration decreased to about $1 \mathrm{sec}$ (Fig. 12D). In contrast to the effects on the duration of excitation and inhibition, we found little change in the cycle period or its profile throughout the episode (see also Fig. 13). These findings indicate that severe medial and dorsal lesions predominantly affect the control of inhibitory and excitatory drive to SART and FEM motoneurons and exert less influence on the production of rhythmic activity. Thus, the pattern or phasing of activity, which is determined by the duration of SART inhibition and FEM excitation, is controlled independently of cycle timing. These findings, together with the observation that normal activity persists in the ventrolateral quadrant isolation, suggest that the neural elements controlling SART inhibition and FEM excita- 
Figure 12. Quantitative comparison of the FEM and SART burst duration and cycle interval from intact T7-L4 cord segments $(A)$ and after various lesions ( $B$, moderate lesions; $C$, severe medial lesions; $D$, severe dorsal lesions). The FEM (open circles) and SART (solid circles) burst duration, SART inhibitory period (open triangles), and the cycle duration (solid triangles) were measured as described in Materials and Methods (Fig. 1B). Each symbol and error bar represents the mean and the SEM pooled from several preparations as indicated to the right of each graph. Drawings to the right of the graphs illustrate the extent of the various lesions. The dotted outline shows the region of cord that was removed, whereas the continuous outline indicates the portion that remained and was recorded from. The straight lines define the level of the section. The arrows in the drawing in $B$ identify two experiments in which medial and dorsal lesions were combined to produce a ventrolateral quadrant isolation. $E$, Percentage changes in burst duration and cycle parameters after various lesions. Since some parameters showed a progressive change during the episode, percentage changes within each preparation were first determined from parameters belonging to the same cycle number of an episode. These percentage changes were then pooled, except for data from the initial cycle, and plotted together with the SEM in the histogram. The asterisks indicate the changes that were significant using the $t$ test $(p<0.01)$. See text for details.
A

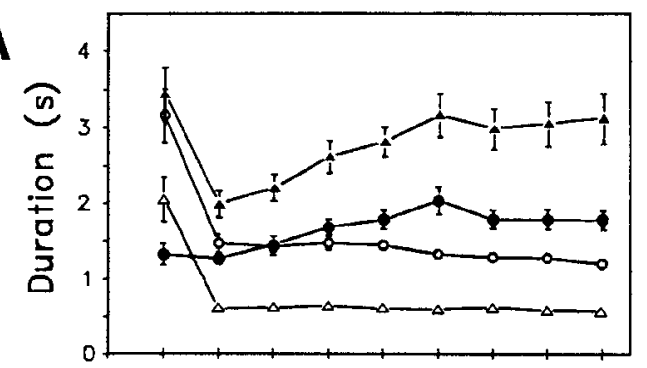

B

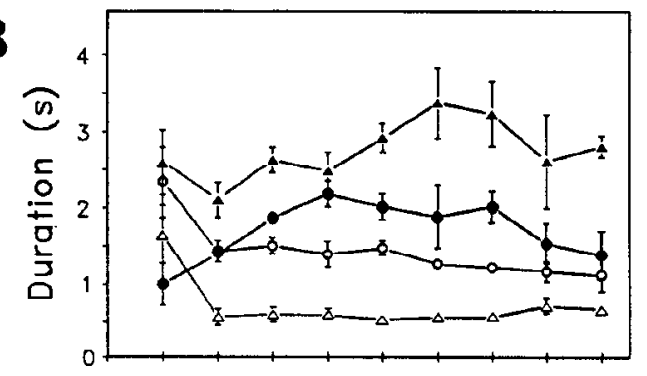

C

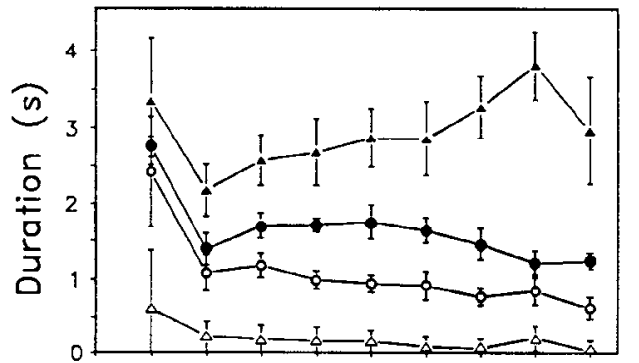

prep $=4$

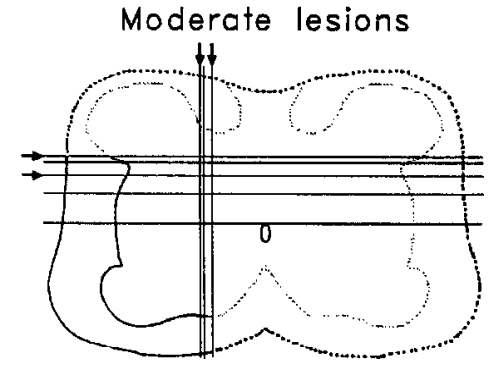

Severe medial lesions

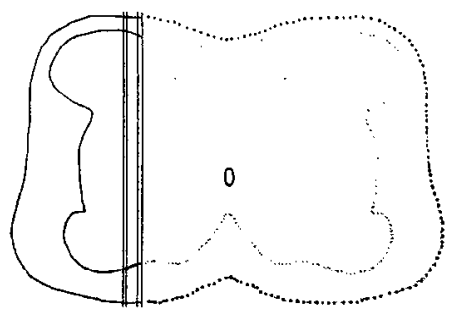

D

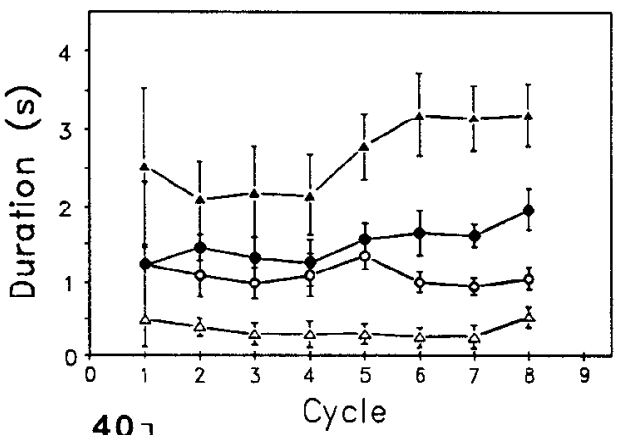

prep $=3$
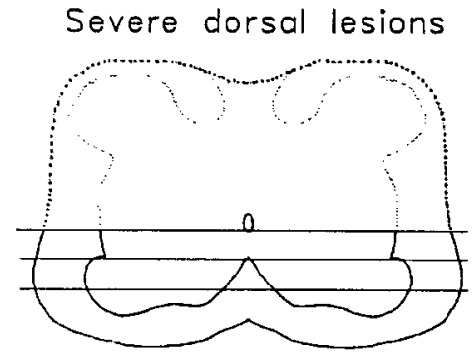

E

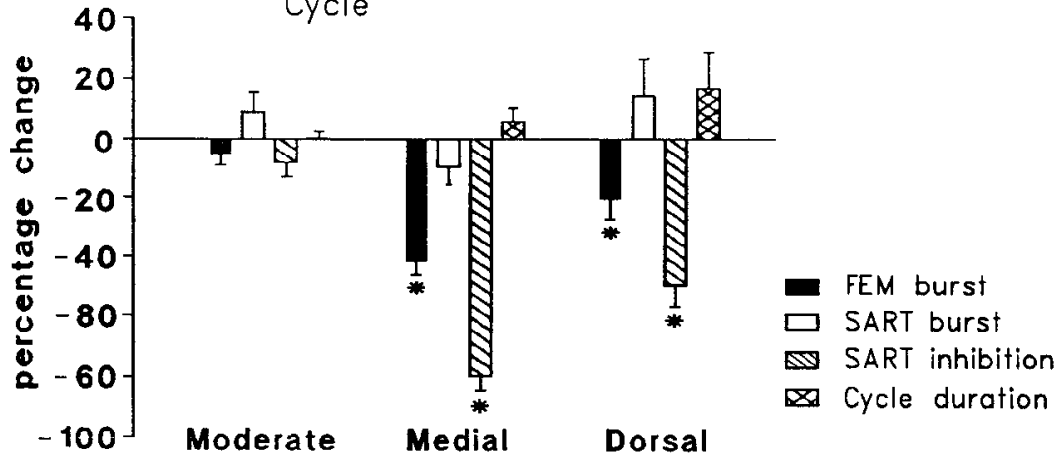

tion are located close to, and just dorsomedial to, the LMC. If this region is preserved, then normal phasing of SART and FEM activity is found. If it is disrupted, by either a dorsal or a medial lesion, then alternation disappears and synchrony results.

The percentage changes in burst and cycle parameters, after excluding data from the initial cycle, are summarized in Figure $12 E$. Since some parameters showed progressive changes during the episode, they were compared for the same cycle number, before and after the lesion, and then pooled for all cycles in the episode and across preparations. We observed statistically significant changes $(p<0.01$ ) only for the FEM burst duration and the SART inhibition following severe dorsal or medial lesions. Although some changes in the SART burst duration did occur, these were smaller than for the other parameters and 

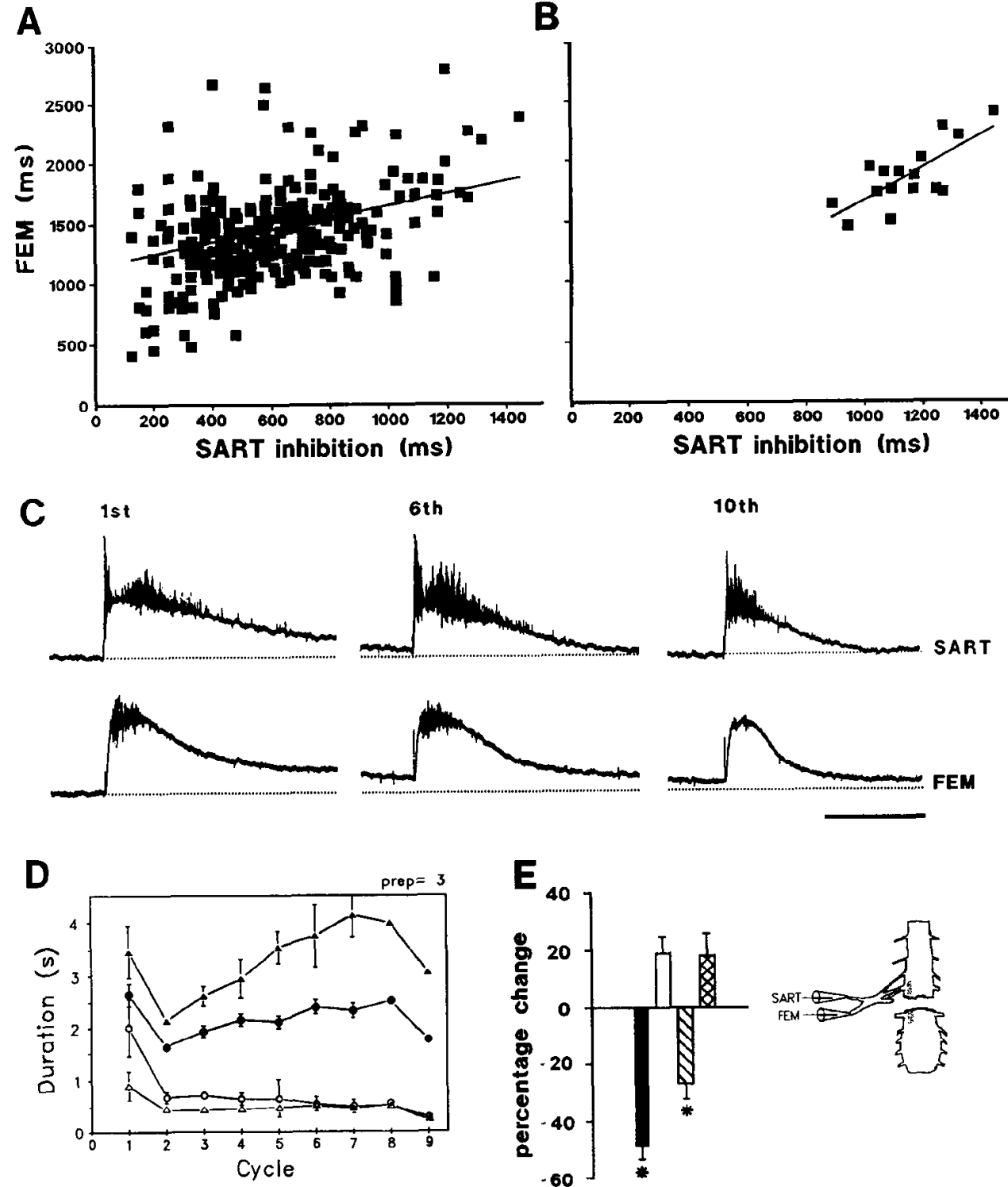

Figure 13. The excitation of FEM motoneurons is correlated with the inhibition of $S A R T$ motoneurons. $A$, Scatter plot of the $F E M$ burst duration and $S A R T$ inhibitory period pooled from 12 control preparations. The coefficient of correlation $(r)$ calculated from these values was 0.31 . $B$, Scatter plot of the $F E M$ burst duration and $S A R T$ inhibitory period in one preparation. The coefficient of correlation was 0.74 in this experiment. $C$, Correlated depression of $S A R T$ inhibition and FEM excitation during repetitive stimulation of the spinal cord. Repetitive stimulation of the cord repeatedly evokes a single cycle in which the timing of $S A R T$ and $F E M$ activity is the initially the same as during rhythmic bursting ( 1 st cycle). With successive stimuli the $S A R T$ inhibition and the $F E M$ burst progressively decline (compare with results of severe medial lesion, Fig. $10 B$ ). $D$, Graphs of cycle parameters (defined as in Fig. 12A) following removal of the caudal part of the cord (LS3-LS9) as illustrated to the right of $E$. $E$, Histogram summarizing the percentage change in the cycle parameters averaged for three caudal lesions. Only the $S A R T$ inhibition and the $F E M$ excitation exhibited statistically significant changes. were not statistically significant. Moreover, in contrast to the FEM burst duration and SART inhibitory period, which remained coupled and fell after the lesions (see also Fig. 13E), the SART burst duration could either decrease or increase, suggesting that it is controlled independently.

\section{Correlation between FEM excitation and SART inhibition}

To investigate the coupling between the FEM excitation and the SART inhibition more fully, we examined the correlation between the duration of the FEM discharge and the SART inhibition under various conditions. Figure $13 A$ illustrates the corrclation between the FEM burst duration and SART inhibitory period in normal cycles of unlesioned animals. The correlation coefficient pooled from 12 control preparations was 0.31 and was significantly different from 0 . This comparatively low correlation coefficient probably resulted from the pooling of data from different embryos because correlations were higher in individual experiments. In $60 \%$ of individual experiments these variables were strongly corrclatcd $(r>0.7)$, as illustrated in Figure $13 B$.
Another example of the coupled behavior of the SART inhibition and the FEM discharge is illustrated in Figure $13 C$, which shows the effect of repetitive cord stimulation on the phasing of activity in SART and FEM motoneurons. The tracings are wideband recordings in which neural discharge is superimposed on a slow depolarization (see also Figs. $2 B, 3,5$, 6). During repetitive stimulation there is a progressive and coupled depression of the SART inhibitory period and the FEM excitation. In the last cycle of the series (10th in Fig. 13C), the SART inhibition has disappeared together with the FEM discharge, although a subthreshold depolarization does persist in the FEM nerve.

Such correlations could arise because the SART inhibition and the FEM excitation are driven by a common source, or alternatively because the SART inhibition is driven by the activity of FEM and other extensor motoneurons through recurrent inhibitory connections from extensors to flexors. In initial experiments we tested this idea by stimulating the FEM nerve antidromically (dorsal roots cut) and recording the effects on SART discharge during rhythmic activity, but we did not detect 
an increase the duration of the inhibitory period of the SART discharge. However, this negative finding may have occurred because of inadequate antidromic invasion of axon collaterals or alternatively, because only a single extensor (FEM) nerve was activated. Therefore, we examined the effects of deleting extensor motoneurons, including part of the FEM motoneuron pool, without affecting SART motoneurons. This was accomplished by removing LS3 (containing 40-50\% of the FEM motoneurons; Landmesser, 1978) together with more caudal segments and then recording the remaining activity in a piece of cord comprising segments T7-LS2 (Fig. 13D,E). This procedure severely reduced the duration of the FEM bursts to $<0.5 \mathrm{sec}$, from control values of 1.3-1.5 sec. Coupled with this decrease in the duration of FEM excitation was a reduction in the duration of SART inhibition (26\%), although this was much smaller than the effects on the FEM excitation. Thus, while this result is compatible with a direct inhibitory effect of extensor activity on the SART inhibition, the effect was small, and the result is complicated by the removal of synaptic inputs from the deleted caudal segments.

\section{Discussion}

Rostrocaudal organization of rhythm-generating networks

Our experiments have revealed that the capacity for rhythmic activity is distributed along the spinal cord and may be functional within a single segment. These results are consistent with studies in other vertebrates that show that rhythm-generating networks are distributed along the cord but may also be functional in one to two segments (Grillner, 1974; Cohen and Wallen, 1980; Deliagina et al., 1983; Cohen, 1987a,b; Mortin and Stein, 1989). We have found, however, that the rhythmogenic capacity of the rostral $40 \%$ of the lumbosacral cord is significantly greater than that of the caudal part. Isolated caudal segments produccd substantially fewer cycles than rostral segments even when NMDA was used as an excitant. A similar rostrocaudal specialization of the hindlimb enlargement has been documented for scratching in the cat (Deliagina et al., 1983; Arshavsky et al., 1984; Gelfand et al., 1988) and in the turtle (Mortin and Stein, 1989) and for cat locomotion (Afelt et al., 1973; Grillner and Zangger, 1979), suggesting that this pattern of organization may be a general feature of vertebrate spinal rhythm generators. Moreover, the similarity in the regionalization of rhythmic networks in the embryonic chick cord with that of mature vertebrates suggests that the networks subserving embryonic motor activity are the precursors of locomotor circuitry in the adult animal.

The mechanisms responsible for the difference in the motor output of isolated rostral and caudal segments are unknown. We were able to eliminate the possibility that caudal segments are less mature than the rostral segments, because the rostrocaudal difference in motor output became more, rather than less, pronounced with development (Fig. $7 D$ ). This result suggests that the rostrocaudal specialization develops from a relatively uniform network in which the individual segmental oscillators are more autonomous than later in development.

Another possibility to account for the differing capacity of the rostral and caudal cord is that the rostral cord is morc cxcitablc than the caudal cord. Such a rostrocaudal gradient of excitability could lead to phase lags in the activation of rostral and caudal segments and has been hypothesized to account for rostrocaudal phase lag observed in the lamprey spinal cord (see Grillner and Matsushima, 1991). However, we found no evidence for a rostrocaudal phasc lag in the activation of motoneurons along the lumbosacral cord. In fact, motoneurons were almost synchronously activated with comparatively small differences in their onset of activity (40-50 msec, compared to a burst duration of $1-2 \mathrm{sec}$ ) that were not systematically related to the rostrocaudal position of the motoneurons (see Fig. 3C).

\section{Mechanisms of coordination of motor pools in different segments}

Our experiments have shown that the near-synchronous onset of activity between different motor pools within the lumbosacral cord is mediated primarily by axons traveling in the ventral and lateral white matter tracts and by synaptic connections within the gray matter. Interruption of these tracts alters the shape and reduces the amplitude of the rhythmic ventral root potentials caudal to the lesion and significantly delays the onset of firing in the affected segments. The presence of these tracts is also sufficient to allow caudal segments to produce the same number of cycles as rostral segments. If the caudal segments are isolated, they can only produce two or three cycles compared to eight or nine in intact cords. A similar result has been obtained in studies of intersegmental coordination in the lamprey spinal cord. If the lateral white matter tracts and the cellular gray matter are cut, leaving medial white matter tracts intact, then coordination of ventral root activity across the lesion is lost (Cohen, 1987a). At present, the neurons responsible for the intersegmental coordination of motor activity are unknown. In the lamprey spinal cord there is evidence for short- and long-axoned coordinating fibers that presumably arise from propriospinal interneurons (Rovainen, 1985; Cohen, 1987b). Grillner and Matsushima (1991) have proposed that excitatory interneurons, which project axons into the white matter, may also play a role in intersegmental coordination. However, direct identification of the coordinating neurons in the lamprey spinal cord has not yet been accomplished. In the chick cord the most plausible candidates are also propriospinal interneurons that send their axons into the VLT. In adult and developing pigeon such neurons project their axons into the lateral white matter tracts (Nornes et al., 1980; Necker, 1990), and in the chick propriospinal projections can span up to 10 segments as early as stages 27-29 (Oppenheim et al., 1988). Recently, we have identified a population of interneurons in the chick cord, whose axons project into the VLT. These neurons are located dorsal to the LMC, and some of them are rhythmically active (Ho and O'Donovan, 1991), raising the possibility that they may involved in the intersegmental coordination of motor activity.

\section{Rhythmogenesis and alternation are produced by separate mechanisms}

In the lamprey and Xenopus spinal cords, mutual inhibition of two antagonistic centers has been proposed to play an essential role in generating the rhythm (Kahn and Roberts, 1982; Grillner et al., 1986). In such models the alternation of contralateral motoneurons and rhythmogenesis are generated by a common mechanism. Several lines of evidence suggest that this mechanism is unlikely to operate in the chick cord, either for the alternation of antagonists or for rhythm generation. In the chick cord, alternation between antagonists is a result of phase shift imposed on the flexors by specific inhibitory inputs whereas 
extensors receive a predominantly excitatory synaptic drive during each cycle of activity (O'Donovan, 1989a; Sernagor and O'Donovan, 1991). Thus, alternation is not accomplished by mutual inhibition of flexor and extensor motoneurons, but rather by the asymmetrical distribution of inhibitory inputs to flexors. Second, the duration of flexor inhibition and extensor excitation is largely independent of cycle period, suggesting that the two processes are generated independently (Fig. $12 A$; see also Landmesser and O'Donovan, 1984; O'Donovan and Landmesser, 1987). In addition, previous developmental studies have shown that rhythmic activity emerges earlier in development than alternation (O'Donovan and Landmesser, 1987). Finally, the data presented in this article show that certain lesions, particularly those medial to the LMC, result in abolition of the alternation without substantial effects on cycle timing. Collectively, these results suggest that rhythm generation in the chick cord is unlikely to be produced by mutual inhibition of antagonistic centers, and is generated by a mechanism that differs from that controlling the phasing of motoneuron activity. Under certain conditions a similar dissociation between rhythmogenesis and alternation can be observed in both the lamprey and Xenopus embryo. In both species, rhythmic activity can persist in the absence of reciprocal inhibition. In the lamprey, bathapplied strychnine can block the alternation of contralateral motoneurons but not the rhythm (Cohen and Harris-Warrick, 1984). Similarly, if the Xenopus cord is split into two halves, contralateral alternation is abolished but rhythmic activity persists (Kahn and Roberts, 1982) that is resistant to G $\Lambda \mathrm{BAergic}$ and glycinergic antagonists (Soffe, 1991).

A separation of the rhythm generation and pattern formation has also been proposed for mammalian respiration (Feldman at al., 1989) and for the paw shake response of the cat (Koshland and Smith, 1989). In the isolated neonatal rat cord, rhythmic phrenic nerve activity persists in the presence of $\mathrm{GABA}_{\mathrm{A}}$ and glycine antagonists, suggesting that $\mathrm{Cl}$-mediated inhibition of antagonist centers is not required for the genesis of the respiratory rhythm (Feldman et al., 1989). These findings, together with the results of the present work, suggest that separate mechanisms for rhythm generation and pattern formation may be a general feature of vertebrate rhythm-generating networks.

Flexor inhibition and extensor excitation may be controlled by a common pathway

Our results show that the duration of the SART inhibition and FEM excitation is correlated during spontaneous episodes of motor activity and under various experimental conditions. A similar correlation exists between flexor inhibition and extensor excitation during spontaneous activity of the chick embryo in ovo (Fig. 4 in Landmesser and O'Donovan, 1984). This correlation could be accomplished by several mechanisms (Fig. 14A). The simplest is that the premotor inhibitory interneurons projecting to SART motoneurons are driven by the same source as the FEM excitation. Alternatively, firing of FEM motoneurons could inhibit SART motoneuron activity through a recurrent collateral circuit (dashed line identified by arrow in Fig. 14A). Recurrent inhibition is present in the chick cord at these stages of development, although its distribution to various motoneuron pools is unknown (Velumian, 1984; O'Donovan, 1989b). We attempted to distinguish these possibilities by stimulating the FEM nerve antidromically during rhythmic activity or by ablating part of the FEM motoneuron pool (together with caudal

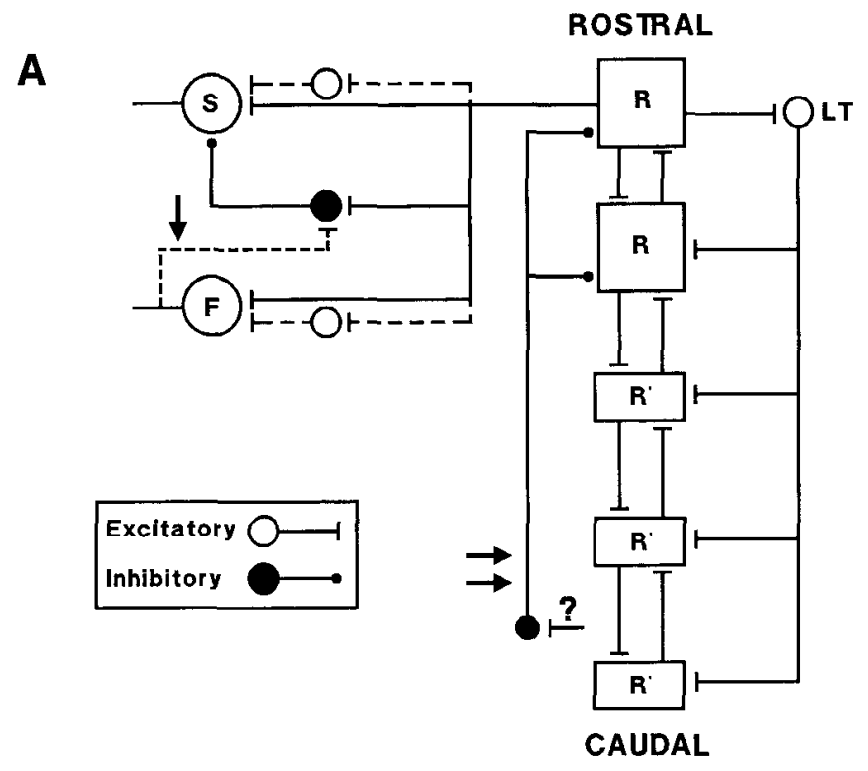

B

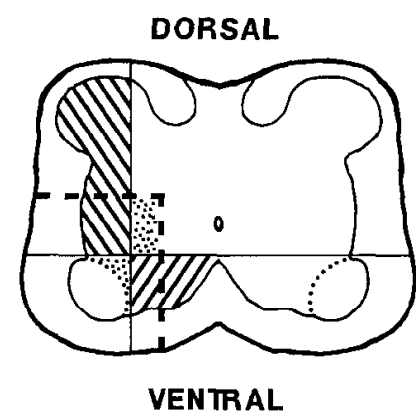

Figure 14. A, Schematic diagram of the central pattern generator in the chick lumbar cord. The rhythm generators $(R)$ are distributed along the rostrocaudal axis of the cord. These generators are coupled by intersegmental projections in the ventrolateral tracts $(L T)$ and by synaptic interactions within the gray matter. With development the more caudal generators $(R)$ become less effective and depend on interactions with the rostral cord. An inhibitory input to the rostral generators is indicated by the double arrows. Its inputs are not determined at present, but they are presumed to arise within the caudal part of the cord. The alternating pattern between SART $(S)$ and FEM $(F)$ motoneurons results from specific inhibition of the SART motoneurons during the initial part of each cycle. The correlation between SART inhibition and FEM burst suggests a common drive to the SART inhibitory (solid circle) and FEM excitatory (open circle) premotor interneurons. The arrowed dashed line indicates an alternative pathway that could account for the correlation between FEM discharge and SART inhibition, but which is less likely (see text). $B$, Map of the regions involved in rhythmogenesis in the chick cord. Rhythmic activity persists in cords with severe dorsal (upper hatching) or medial (lower hatching) lesions, but alternation is depressed or abolished. If the stippled region, dorsomedial to the $\mathrm{LMC}$, is left intact, then normal alternation occurs. This region may contain the cell bodies of the interneurons controlling SART inhibition and FEM excitation.

segments) to reduce the intensity of presumed recurrent inhibition. Antidromic stimulation was without effects on the SART inhibition, and FEM motor pool ablation resulted in a modest change in the duration of SART inhibition $(-26 \%)$ that could easily have resulted from the deletion of premotor interneurons other than those contributing to recurrent inhibition. Thus, although we cannot rule out a role for recurrent inhibition (from 
extensor to flexor motoneurons), our data are more compatible with coexcitation of FEM motoneurons and the inhibitory interneurons projecting to SART motoneurons.

\section{Regionalization of rhythm-generating circuitry in the ventrolateral part of the spinal cord}

The present experiments provide information concerning the location of some of the components of the rhythm and patterngenerating circuitry (Fig. 14B). We found that both the rhythm and the alternation of SART and FEM were preserved in the isolated ventrolateral quadrant of the cord. Thus, networks in this region are sufficient to produce the motor pattern. Demonstrating that such networks are necessary for the expression of alternating motor activity was technically not possible because smaller lesions within the ventrolateral quadrant (to ablate the interneuronal networks) could not be made without compromising the integrity of the motor nuclei.

We also observed rhythmic activity in spinal cords with severe dorsal or medial lesions. In such preparations the only remaining gray matter outside the LMC was a thin lateral or ventral strip. Despite the removal of such large numbers of neurons, the timing of cyclical activity was virtually undisturbed (Fig. 12C,D). These findings suggest two possibilities. If premotor interneurons are responsible for generating the rhythm, then only a small complement of these neurons is required. Furthermore, since the rhythm could be generated by ventral or lateral neurons, the rhythm-generating network does not appear to be localized. The alternative possibility is that the interneurons are located very close to or within the LMC, or that motoneurons themselves participate in the genesis of the rhythm. In other vertebrate preparations, motoneurons have not generally been considered part of the rhythm-generating circuitry (Grillner et al., 1986; Roberts et al., 1986). However, motoneurons can express rhythmic pacemaker-like potentials (Grillner and Wallen, 1985; Durand, 1991), which could contribute to the maintenance of the rhythm. In the chick cord it is unlikely that motoneurons are the sole source of the rhythm because they clearly receive rhythmic synaptic inputs during motor activity (O'Donovan, 1989a; Sernagor and O'Donovan, 1991), which can be depressed by amino acid antagonists (Barry and O'Donovan, 1987), or by reducing extracellular $\mathrm{Ca}^{2+}$ (S. Ho and M. J. O'Donovan, unpublished observations).

An important unresolved question is whether a distinct class of pacemaker cells exists in the chick cord analogous to those proposed for the respiratory system of the neonatal rat (Smith et al., 1991), or whether rhythmicity results from the interaction of neurons individually lacking oscillatory behavior (Friesen et al., 1976). Further studies of ventrally located interneurons will be required to answer these questions.

\section{References}

Afelt Z, Veber NB, Maksimova EV (1973) Reflex activity of chronically isolated spinal cord of the cat. Moscow: Nauka.

Agoston DV, Eiden LE, Brenneman DE (1991) Calcium-dependent regulation of the enkephalin phenotype by neuronal activity during early ontogeny. J Neurosci Res 28:140-148.

Arshavsky YI, Gelfand IM, Orlovsky GN, Pavlova GA, Popova LB (1984) Origin of signals conveyed by the ventral spino-cerebellar tract and spino-reticular-cerebellar pathway. Exp Brain Res 54:426431.

Baev KV (1978) Periodic changes in primary afferent depolarization during fictitious locomotion in thalamic cats. Neurophysiology 10: 316-317.
Barry M, O'Donovan MJ (1987) The effects of excitatory amino acids and their antagonists on the generation of motor activity in the isolated chick cord. Dev Brain Res 36:271-276.

Bekoff A, Stein PSG, Hamburger V (1975) Coordinated motor output in the hindlimb of the 7-day chick embryo. Proc Natl Acad Sci USA 72:1245-1248.

Cohen A (1987a) Effects of oscillator frequency on phase-locking in the lamprey central pattern generator. J Neurosci Methods 21:113125.

Cohen A (1987b) Intersegmental coordinating system of the lamprey central pattern generator for locomotion. J Comp Physiol 160:181193.

Cohen AH, Harris-Warrick RM (1984) Strychnine eliminates alternating motor outputs during fictive locomotion in the lamprey. Brain Res 293:164-167.

Cohen AH, Wallen P (1980) The neuronal correlate of locomotion in fish. "Fictive swimming" induced in an in vitro preparation of the lamprey spinal cord. Exp Brain Res 41:11-18.

Corner MA, Bour HL, Mirmiran M (1979) Development of spontaneous motility and its physiological interpretation in the rat, chick, and frog. In: Neural growth and differentiation (Meisami E, Brazier MA, eds), pp 253-267. New York: Raven.

Deliagina TG, Orlovski GN, Pavlova GA (1983) The capacity for generation of rhythmic oscillations is distributed in the lumbosacral spinal cord of the cat. Exp Brain Res 53:81-90.

Dubuc R, Cabelguen JM, Rossignol S (1985) Rhythmic antidromic discharges of single primary afferents recorded in cut dorsal root filaments during locomotion in the cat. Brain Res 359:375-378.

Durand J (1991) NMDA actions on rat abducens motoneurons. Eur J Neurosci 3:621-633.

Feldman JL, Smith JC (1989) Cellular mechanisms underlying modulation of breathing pattern in mammals. Ann NY Acad Sci 563: 114-130.

Friesen WO, Poon M, Stent GS (1976) An oscillatory neuronal circuit generating a locomotory rhythm. Proc Natl Acad Sci USA 73:3734 3738.

Gelfand IM, Orlovsky GN, Shik M (1988) Locomotion and scratching in tetrapods. In: Neural control of rhythmic movements in vertebrates (Cohen AH, Rossignol S, Grillner S, eds). New York: Wiley.

Grillner S (1974) On the generation of locomotion in the spinal dogfish. Exp Brain Res 20:459-470.

Grillner S, Matsushima T (1991) The neural network underlying locomotion in lamprey-synaptic and cellular mechanisms. Neuron 7:1-15.

Grillner S, Wallen P (1984) How does the lamprey central nervous system make the lamprey swim? J Exp Biol 112:337-357.

Grillner S, Zangger P (1979) On the central generation of locomotion in the low spinal cat. Exp Brain Res 34:241-261.

Grillner S, Brodin L, Sigvardt K, Dalc N (1986) On the spinal network generating locomotion in the lamprey: transmitters, membrane properties and circuitry. In: Neurobiology of vertebrate locomotion, Chap 22 (Grillner S, Stein PSG, Forssberg H, Herman RA, eds). London: Macmillan.

Hamburger V (1973) Anatomical and physiological basis of embryonic motility in birds and mammals. In: Behavioral embryology (Gottlieb G, ed), pp 51-76. New York: Academic.

Hamburger V, Balaban M (1963) Observations and experiments on spontaneous rhythmical behavior in the chick embryo. Dev Biol 7:533545.

Hamburger V, Balaban M, Oppenheim R, Wenger E (1965) Periodic motility of normal and spinal chick embryos between 8 and 17 days of incubation. J Exp Zool 159:1-14.

Hamburger V, Wenger E, Oppenheim R (1966) Motility in the chick embryo in the absence of sensory input. J Exp Zool 162:133-155.

Ho S, O'Donovan MJ (1990) The effects of selective ablations on the rhythmic motor output of the isolated chick spinal cord. Soc Neurosci Abstr 16:114.

Ho S, O'Donovan MJ (1991) Properties of propriospinal neurons involved in the rhythmic excitation of motor pools in the isolated embryonic chick spinal cord. Soc Neurosci Abstr 17:120.

Hollyday M, Hamburger V (1977) An autoradiographic study of the formation of the lateral motor column in the chick embryo. Brain Res 132:197-208.

Kahn JA, Roberts A (1982) Experiments on the central pattern generator for swimming in amphibian embryos. Philos Trans R Soc Lond [Biol] 296:229-243. 
Landmesser L (1978) The distribution of motoneurons supplying chick hind limb muscles. J Physiol (Lond) 284:371-389.

Landmesser LT, O'Donovan MJ (1984) Activation patterns of embryonic chick hindlimb muscles recorded in-ovo and in an isolated spinal cord preparation. J Physiol (Lond) 347:189-204.

Mattson MP (1988) Neurotransmitters in the regulation of neuronal cytoarchitecture. Brain Res Rev 13:179-212.

Mattson MP, Hauscr KF (1991) Spatial and temporal integration of neurotransmitter signals in the development of neural circuitry. Neurochem Int 19:17-24.

McLennan I (1983) Neural dependence and independence of myotube production in chicken hindlimb muscles. Dev Biol 98:287-294.

Meier E, Hertz L, Schousboe A (1991) Neurotransmitters as developmental signals. Neurochem Int 19:1-15.

Mortin LI, Stein PSG (1989) Spinal cord segments containing key elements of the central pattern generators for three forms of scratch reflex in the turtle. J Neurosci 9:2285-2296.

Necker R (1990) Cells of origin of ascending and descending as well as branching fibers in the cervical spinal cord of the pigeon. Neurosci Lett 119:1-4.

Nornes HO, Hart H, Carry M (1980) Pattern of development of ascending and descending fibers in embryonic spinal cord of chick. 1. Role of positional 'information.' J Comp Neurol 192:119-132.

O'Donovan MJ (1989a) Motor activity in the isolated spinal cord of the chick embryo: synaptic drive and firing pattern of single motoneurons. J Neurosci 9:943-958.

O'Donovan MJ (1989b) The development of recurrent motoneuronal connections in the spinal cord of the chick embryo. Soc Neurosci Abstr 15:919.

O'Donovan MJ, Landmesser LT (1987) The development of hindlimb motor activity studied in an isolated preparation of the chick spinal cord. J Neurosci 7:3256-3264.

O'Donovan MJ, Sernagor E, Sholomenko G, Ho S, Antal M, Yee W (1992) Development of spinal motor networks in the chick embryo. J Exp Zool 261:261-273.

Oppenheim RW (1975) The role of supraspinal input in embryonic motility: a re-examination in the chick. J Comp Neurol 160:37-50.

Oppenheim RW, Shneiderman A, Shimizu I, Yaginuma H (1988) Onset and development of intersegmental projections in the chick embryo spinal cord. J Comp Neurol 275:159-180.

Pittman R, Oppenheim RN (1978) Neuromuscular blockade increases motoneuron survival during normal cell death in the chick embryo. Nature 271:364-366.
Provine RR (1971) Embryonic spinal cord: synchrony and spatial distribution of polyneuronal burst discharges. Brain Res 29:155-158.

Provine RR, Rogers L (1977) Development of spinal cord bioelectric activity in spinal chick embryos and its behavioral consequences. $J$ Neurobiol 8:217-228.

Provine RR, Sharma SC, Sandel TT, Hamburger V (1970) Electrical activity in the spinal cord of the chick embryo, in situ. Proc Natl Acad Sci USA 65:508-515.

Ripley KL, Provine RR (1972) Neural correlates of embryonic motility in the chick. Brain Res 45:127-134.

Roberts A, Soffe SR, Dale N (1986) Spinal interneurones and swimming in frog embryos. In: Neurobiology of vertebrate locomotion, Chap 19 (Grillner S, Stein PSG, Stuart DG, Forssberg H, Herman RM, eds). London: Macmillan.

Rovainen CM (1985) Effects of groups of propriospinal interneurons on fictive swimming in the isolated spinal cord of the lamprey. $J$ Neurophysiol 54:959-977.

Russell DF, Wallen P (1980) On the pattern generator for fictive swimming in the lamprey, Ichthyomyzon unicuspis. Acta Physiol Scand 108:9A.

Sernagor E, O'Donovan MJ (1991) Whole cell patch clamp of rhythmically active neurons in the isolated spinal cord of the chick embryo. Neurosci Lett 128:221-216.

Sharma SC, Provine RR, Hamburger V, Sandel T (1970) Unit activity in the isolated spinal cord of the chick embryo. Proc Natl Acad Sci USA $66: 40-47$.

Smith JC, Ellenberger HH, Ballanyi K, Richter DW, Feldman JL (1991) Pre-Botzinger complex: a brainstem region that may generate respiratory rhythm in mammals. Science 254:726-729.

Soffe SR (1989) Roles of glycinergic inhibition and $N$-methyl-D-aspartate receptor mediated excitation in the locomotor rhythmicity of one half of the Xenopus embryo central nervous system. Eur J Neurosci 1:561-571.

Srihari T, Vrbova G (1978) The role of muscle activity in the differentiation of neuromuscular junctions in slow and fast chick muscles. J Neurocytol 7:529-540.

Velumian AA (1984) Direct evidence for postsynaptic inhibition in the embryonic chick spinal cord. Dev Brain Res 14:229-239.

Williams C, Wholenberg G, O'Donovan MJ (1987) Regional variations in the extent and timing of motoneuron cell death in the lumbosacral spinal cord of the chick embryo. Dev Brain Res 34:215-221. 